\title{
Hochschild Homology and Cohomology of Klein Surfaces ${ }^{\star}$
}

\author{
Frédéric BUTIN
}

Université de Lyon, Université Lyon 1, CNRS, UMR5208, Institut Camille Jordan, 43 blvd du 11 novembre 1918, F-69622 Villeurbanne-Cedex, France

E-mail: butin@math.univ-lyon1.fr

URL: http://math.univ-lyon1.fr/ butin/

Received April 09, 2008, in final form September 04, 2008; Published online September 17, 2008

Original article is available at http://www.emis.de/journals/SIGMA/2008/064/

\begin{abstract}
Within the framework of deformation quantization, a first step towards the study of star-products is the calculation of Hochschild cohomology. The aim of this article is precisely to determine the Hochschild homology and cohomology in two cases of algebraic varieties. On the one hand, we consider singular curves of the plane; here we recover, in a different way, a result proved by Fronsdal and make it more precise. On the other hand, we are interested in Klein surfaces. The use of a complex suggested by Kontsevich and the help of Groebner bases allow us to solve the problem.
\end{abstract}

Key words: Hochschild cohomology; Hochschild homology; Klein surfaces; Groebner bases; quantization; star-products

2000 Mathematics Subject Classification: 53D55; 13D03; 30F50; 13P10

\section{Introduction}

\subsection{Deformation quantization}

Given a mechanical system $(M, \mathcal{F}(M))$, where $M$ is a Poisson manifold and $\mathcal{F}(M)$ the algebra of regular functions on $M$, it is important to be able to quantize it, in order to obtain more precise results than through classical mechanics. An available method is deformation quantization, which consists of constructing a star-product on the algebra of formal power series $\mathcal{F}(M)[[\hbar]]$. The first approach for this construction is the computation of Hochschild cohomology of $\mathcal{F}(M)$.

We consider such a mechanical system given by a Poisson manifold $M$, endowed with a Poisson bracket $\{\cdot, \cdot\}$. In classical mechanics, we study the (commutative) algebra $\mathcal{F}(M)$ of regular functions (i.e., for example, $\mathcal{C}^{\infty}$, holomorphic or polynomial) on $M$, that is to say the observables of the classical system. But quantum mechanics, where the physical system is described by a (non commutative) algebra of operators on a Hilbert space, gives more correct results than its classical analogue. Hence the importance to get a quantum description of the classical system $(M, \mathcal{F}(M))$, such an operation is called a quantization.

One option is geometric quantization, which allows us to construct in an explicit way a Hilbert space and an algebra of operators on this space (see the book [10] on the Virasoro group and algebra for a nice introduction to geometric quantization). This very interesting method presents the drawback of being seldom applicable.

That is why other methods, such as asymptotic quantization and deformation quantization, have been introduced. The latter, described in 1978 by F. Bayen, M. Flato, C. Fronsdal, A. Lichnerowicz and D. Sternheimer in [5], is a good alternative: instead of constructing an

\footnotetext{
${ }^{\star}$ This paper is a contribution to the Special Issue on Deformation Quantization. The full collection is available at http://www.emis.de/journals/SIGMA/Deformation_Quantization.html
} 
algebra of operators on a Hilbert space, we define a formal deformation of $\mathcal{F}(M)$. This is given by the algebra of formal power series $\mathcal{F}(M)[[\hbar]]$, endowed with some associative, but not always commutative, star-product,

$$
f * g=\sum_{j=0}^{\infty} m_{j}(f, g) \hbar^{j},
$$

where the maps $m_{j}$ are bilinear and where $m_{0}(f, g)=f g$. Then quantization is given by the map $f \mapsto \widehat{f}$, where the operator $\widehat{f}$ satisfies $\widehat{f}(g)=f * g$.

In which cases does a Poisson manifold admit such a quantization? The answer was given by Kontsevich in [11]: in fact he constructed a star-product on every Poisson manifold. Besides, he proved that if $M$ is a smooth manifold, then the equivalence classes of formal deformations of the zero Poisson bracket are in bijection with equivalence classes of star-products. Moreover, as a consequence of the Hochschild-Kostant-Rosenberg theorem, every Abelian star-product is equivalent to a trivial one.

In the case where $M$ is a singular algebraic variety, say

$$
M=\left\{\mathbf{z} \in \mathbb{C}^{n} / f(\mathbf{z})=0\right\},
$$

with $n=2$ or 3 , where $f$ belongs to $\mathbb{C}[\mathbf{z}]$ - and this is the case which we shall study - we shall consider the algebra of functions on $M$, i.e. the quotient algebra $\mathbb{C}[\mathbf{z}] /\langle f\rangle$. So the above mentioned result is not always valid. However, the deformations of the algebra $\mathcal{F}(M)$, defined by the formula (1), are always classified by the Hochschild cohomology of $\mathcal{F}(M)$, and we are led to the study of the Hochschild cohomology of $\mathbb{C}[\mathbf{z}] /\langle f\rangle$.

\subsection{Cohomologies and quotients of polynomial algebras}

We shall now consider $R:=\mathbb{C}\left[z_{1}, \ldots, z_{n}\right]=\mathbb{C}[\mathbf{z}]$, the algebra of polynomials in $n$ variables with complex coefficients. We also fix $m$ elements $f_{1}, \ldots, f_{m}$ of $R$, and we define the quotient algebra $A:=R /\left\langle f_{1}, \ldots, f_{m}\right\rangle=\mathbb{C}\left[z_{1}, \ldots, z_{n}\right] /\left\langle f_{1}, \ldots, f_{m}\right\rangle$.

Recent articles were devoted to the study of particular cases, for Hochschild as well as for Poisson homology and cohomology:

C. Roger and P. Vanhaecke, in [16], calculate the Poisson cohomology of the affine plane $\mathbb{C}^{2}$, endowed with the Poisson bracket $f_{1} \partial_{z_{1}} \wedge \partial_{z_{2}}$, where $f_{1}$ is a homogeneous polynomial. They express it in terms of the number of irreducible components of the singular locus $\left\{\mathbf{z} \in \mathbb{C}^{2} / f_{1}(\mathbf{z})=0\right\}$ (in this case, we have a symplectic structure outside the singular locus), the algebra of regular functions on this curve being the quotient algebra $\mathbb{C}\left[z_{1}, z_{2}\right] /\langle f\rangle$.

M. Van den Bergh and A. Pichereau, in [18, 13] and [14], are interested in the case where $n=3$ and $m=1$, and where $f_{1}$ is a weighted homogeneous polynomial with an isolated singularity at the origin. They compute the Poisson homology and cohomology, which in particular may be expressed in terms of the Milnor number of the space $\mathbb{C}\left[z_{1}, z_{2}, z_{3}\right] /\left\langle\partial_{z_{1}} f_{1}, \partial_{z_{2}} f_{1}, \partial_{z_{3}} f_{1}\right\rangle$ (the definition of this number is given in [3]).

Once more in the case where $n=3$ and $m=1$, in [2], J. Alev and T. Lambre compare the Poisson homology in degree 0 of Klein surfaces with the Hochschild homology in degree 0 of $A_{1}(\mathbb{C})^{G}$, where $A_{1}(\mathbb{C})$ is the Weyl algebra and $G$ the group associated to the Klein surface. We shall give more details about those surfaces in Section 4.1.

In [1], J. Alev, M.A. Farinati, Th. Lambre and A.L. Solotar establish a fundamental result: they compute all the Hochschild homology and cohomology spaces of $A_{n}(\mathbb{C})^{G}$, where $A_{n}(\mathbb{C})$ 
is the Weyl algebra, for every finite $\operatorname{subgroup} G$ of $\mathbf{S p}_{2 n} \mathbb{C}$. It is an interesting and classical question to compare the Hochschild homology and cohomology of $A_{n}(\mathbb{C})^{G}$ with the Poisson homology and cohomology of the ring of invariants $\mathbb{C}[\mathbf{x}, \mathbf{y}]^{G}$, which is a quotient algebra of the form $\mathbb{C}[\mathbf{z}] /\left\langle f_{1}, \ldots, f_{m}\right\rangle$.

C. Fronsdal studies in [8] Hochschild homology and cohomology in two particular cases: the case where $n=1$ and $m=1$, and the case where $n=2$ and $m=1$. Besides, the appendix of this article gives another way to calculate the Hochschild cohomology in the more general case of complete intersections.

In this paper, we propose to calculate the Hochschild homology and cohomology in two particularly important cases.

- The case of singular curves of the plane, with polynomials $f_{1}$ which are weighted homogeneous polynomials with a singularity of modality zero: these polynomials correspond to the normal forms of weighted homogeneous functions of two variables and of modality zero, given in the classification of weighted homogeneous functions of [3] (this case already held C. Fronsdal's attention).

- The case of Klein surfaces $\mathcal{X}_{\Gamma}$ which are the quotients $\mathbb{C}^{2} / \Gamma$, where $\Gamma$ is a finite subgroup of $\mathbf{S L}_{2} \mathbb{C}$ (this case corresponds to $n=3$ and $m=1$ ). The latter have been the subject of many works; their link with the finite subgroups of $\mathbf{S L}_{2} \mathbb{C}$, with the Platonic polyhedra, and with McKay correspondence explains this large interest. Moreover, the preprojective algebras, to which [6] is devoted, constitute a family of deformations of the Klein surfaces, parametrized by the group which is associated to them: this fact justifies once again the calculation of their cohomology.

The main result of the article is given by two propositions:

Proposition 1. Given a singular curve of the plane, defined by a polynomial $f \in \mathbb{C}[\mathbf{z}]$, of type $A_{k}, D_{k}$ or $E_{k}$. For $j \in \mathbb{N}$, let $H H^{j}$ (resp. $H H_{j}$ ) be the Hochschild cohomology (resp. homology) space in degree $j$ of $A:=\mathbb{C}[\mathbf{z}] /\langle f\rangle$, and let $\nabla f$ be the gradient of $f$. Then $H H^{0} \simeq$ $H H_{0} \simeq A, H H^{1} \simeq A \oplus \mathbb{C}^{k}$ and $H H_{1} \simeq A^{2} /(A \nabla f)$, and for all $j \geq 2, H H^{j} \simeq H H_{j} \simeq \mathbb{C}^{k}$.

Proposition 2. Let $\Gamma$ be a finite subgroup of $\mathbf{S L}_{2} \mathbb{C}$ and $f \in \mathbb{C}[\mathbf{z}]$ such that $\mathbb{C}[x, y]^{\Gamma} \simeq \mathbb{C}[\mathbf{z}] /\langle f\rangle$. For $j \in \mathbb{N}$, let $H H^{j}$ (resp. $H H_{j}$ ) be the Hochschild cohomology (resp. homology) space in degree $j$ of $A:=\mathbb{C}[\mathbf{z}] /\langle f\rangle$, and let $\nabla f$ be the gradient of $f$. Then $H H^{0} \simeq H H_{0} \simeq A$, $H H^{1} \simeq(\nabla f \wedge$ $\left.A^{3}\right) \oplus \mathbb{C}^{\mu}$ and $H H_{1} \simeq \nabla f \wedge A^{3}, H H^{2} \simeq A \oplus \mathbb{C}^{\mu}$ and $H H_{2} \simeq A^{3} /\left(\nabla f \wedge A^{3}\right)$, and for all $j \geq 3$, $H H^{j} \simeq H H_{j} \simeq \mathbb{C}^{\mu}$, where $\mu$ is the Milnor number of $\mathcal{X}_{\Gamma}$.

For explicit computations, we shall make use of, and develop a method suggested by M. Kontsevich in the appendix of [8].

We will first study the case of singular curves of the plane in Section 3: we will use this method to recover the result that C. Fronsdal proved by direct calculations. Then we will refine it by determining the dimensions of the cohomology and homology spaces by means of multivariate division and Groebner bases.

Next, in Section 4, we will consider the case of Klein surfaces $\mathcal{X}_{\Gamma}$. For $j \in \mathbb{N}$, we denote by $H H^{j}$ the Hochschild cohomology space in degree $j$ of $\mathcal{X}_{\Gamma}$. We will first prove that $H H^{0}$ identifies with the space of polynomial functions on the singular surface $\mathcal{X}_{\Gamma}$. We will then prove that $H H^{1}$ and $H H^{2}$ are infinite-dimensional. We will also determine, for $j$ greater or equal to 3 , the dimension of $H H^{j}$, by showing that it is equal to the Milnor number of the surface $\mathcal{X}_{\Gamma}$. Finally, we will compute the Hochschild homology spaces.

In Section 1.3 we begin by recalling important classical results about deformations. 


\subsection{Hochschild homology and cohomology and deformations of algebras}

Consider an associative $\mathbb{C}$-algebra, denoted by $A$. The Hochschild cohomological complex of $A$ is

$$
C^{0}(A) \stackrel{d^{(0)}}{\longrightarrow} C^{1}(A) \stackrel{d^{(1)}}{\longrightarrow} C^{2}(A) \stackrel{d^{(2)}}{\longrightarrow} C^{3}(A) \stackrel{d^{(3)}}{\longrightarrow} C^{4}(A) \stackrel{d^{(4)}}{\longrightarrow} \cdots
$$

where the space $C^{p}(A)$ of $p$-cochains is defined by $C^{p}(A)=0$ for $p \in-\mathbb{N}^{*}, C^{0}(A)=A$, and for $p \in \mathbb{N}^{*}, C^{p}(A)$ is the space of $\mathbb{C}$-linear maps from $A^{\otimes p}$ to $A$. The differential $d=\bigoplus_{i=0}^{\infty} d^{(p)}$ is given by

$$
\begin{aligned}
\forall f \in C^{p}(A), \quad & d^{(p)} f\left(a_{0}, \ldots, a_{p}\right)=a_{0} f\left(a_{1}, \ldots, a_{p}\right) \\
& -\sum_{i=0}^{p-1}(-1)^{i} f\left(a_{0}, \ldots, a_{i} a_{i+1}, \ldots, a_{p}\right)+(-1)^{p} f\left(a_{0}, \ldots, a_{p-1}\right) a_{p} .
\end{aligned}
$$

We may write it in terms of the Gerstenhaber bracket $^{1}[\cdot, \cdot]_{G}$ and of the product $\mu$ of $A$, as follows

$$
d^{(p)} f=(-1)^{p+1}[\mu, f]_{G} .
$$

Then we define the Hochschild cohomology of $A$ as the cohomology of the Hochschild cohomological complex associated to $A$, i.e. $H H^{0}(A):=\operatorname{Ker} d^{(0)}$ and for $p \in \mathbb{N}^{*}, H H^{p}(A):=$ $\operatorname{Ker} d^{(p)} / \operatorname{Im} d^{(p-1)}$.

We denote by $\mathbb{C}[[\hbar]]$ (resp. $A[[\hbar]])$ the algebra of formal power series in the parameter $\hbar$, with coefficients in $\mathbb{C}$ (resp. $A$ ). A deformation of the map $\mu$ is a map $m$ from $A[[\hbar]] \times A[[\hbar]]$ to $A[[\hbar]]$ which is $\mathbb{C}[[\hbar]]$-bilinear and such that

$$
\begin{aligned}
& \forall(s, t) \in A[[\hbar]]^{2}, \quad m(s, t)=s t \quad \bmod \hbar A[[\hbar]], \\
& \forall(s, t, u) \in A[[\hbar]]^{3}, \quad m(s, m(t, u))=m(m(s, t), u) .
\end{aligned}
$$

This means that there exists a sequence of bilinear maps $m_{j}$ from $A \times A$ to $A$ of which the first term $m_{0}$ is the product of $A$ and such that

$$
\begin{aligned}
& \forall(a, b) \in A^{2}, \quad m(a, b)=\sum_{j=0}^{\infty} m_{j}(a, b) \hbar^{j}, \\
& \forall n \in \mathbb{N}, \quad \sum_{i+j=n} m_{i}\left(a, m_{j}(b, c)\right)=\sum_{i+j=n} m_{i}\left(m_{j}(a, b), c\right), \\
& \text { that is to say } \sum_{i+j=n}\left[m_{i}, m_{j}\right]_{G}=0 .
\end{aligned}
$$

We say that $(A[[\hbar]], m)$ is a deformation of the algebra $(A, \mu)$. We say that the deformation is of order $p$ if the previous formulae are satisfied (only) for $n \leq p$.

The Hochschild cohomology plays an important role in the study of deformations of the algebra $A$, by helping us to classify them. In fact, if $\pi \in C^{2}(A)$, we may construct a first order deformation $m$ of $A$ such that $m_{1}=\pi$ if and only if $\pi \in \operatorname{Ker} d^{(2)}$. Moreover, two first order

\footnotetext{
${ }^{1}$ Recall that for $F \in C^{p}(A)$ and $H \in C^{q}(A)$, the Gerstenhaber product is the element $F \bullet H \in C^{p+q-1}(A)$ defined by $F \bullet H\left(a_{1}, \ldots, a_{p+q-1}\right)=\sum_{i=0}^{p-1}(-1)^{i(q+1)} F\left(a_{1}, \ldots, a_{i}, H\left(a_{i+1}, \ldots, a_{i+q}\right), a_{i+q+1}, \ldots, a_{p+q-1}\right)$, and the Gerstenhaber bracket is $[F, H]_{G}:=F \bullet H-(-1)^{(p-1)(q-1)} H \bullet F$. See for example [9], and [4, page 38].
} 
deformations are equivalent ${ }^{2}$ if and only if their difference is an element of $\operatorname{Im} d^{(1)}$. So the set of equivalence classes of first order deformations is in bijection with $H H^{2}(A)$.

If $m=\sum_{j=0}^{p} m_{j} \hbar^{j}, m_{j} \in C^{2}(A)$ is a deformation of order $p$, then we may extend $m$ to a deformation of order $p+1$ if and only if there exists $m_{p+1}$ such that

$$
\begin{aligned}
& \forall(a, b, c) \in A^{3}, \quad \sum_{i=1}^{p}\left(m_{i}\left(a, m_{p+1-i}(b, c)\right)-m_{i}\left(m_{p+1-i}(a, b), c\right)\right)=-d^{(2)} m_{p+1}(a, b, c), \\
& \text { i.e. } \quad \sum_{i=1}^{p}\left[m_{i}, m_{p+1-i}\right]_{G}=2 d^{(2)} m_{p+1} .
\end{aligned}
$$

According to the graded Jacobi identity for $[\cdot, \cdot]_{G}$, the last sum belongs to $\operatorname{Ker} d^{(3)}$. So $H H^{3}(A)$ contains the obstructions to extend a deformation of order $p$ to a deformation of order $p+1$.

The Hochschild homological complex of $A$ is

$$
\cdots \stackrel{d_{5}}{\longrightarrow} C_{4}(A) \stackrel{d_{4}}{\longrightarrow} C_{3}(A) \stackrel{d_{3}}{\longrightarrow} C_{2}(A) \stackrel{d_{2}}{\longrightarrow} C_{1}(A) \stackrel{d_{1}}{\longrightarrow} C_{0}(A)
$$

where the space of $p$-chains is given by $C_{p}(A)=0$ for $p \in-\mathbb{N}^{*}, C_{0}(A)=A$, and for $p \in \mathbb{N}^{*}$, $C_{p}(A)=A \otimes A^{\otimes p}$. The differential $d=\bigoplus_{i=0}^{\infty} d_{p}$ is given by

$$
\begin{aligned}
& d_{p}\left(a_{0} \otimes a_{1} \otimes \cdots \otimes a_{p}\right)=a_{0} a_{1} \otimes a_{2} \otimes \cdots \otimes a_{p} \\
& \quad+\sum_{i=1}^{p-1}(-1)^{i} a_{0} \otimes a_{1} \otimes \cdots \otimes a_{i} a_{i+1} \otimes \cdots \otimes a_{p}+(-1)^{p} a_{p} a_{0} \otimes a_{1} \otimes \cdots \otimes a_{p-1} .
\end{aligned}
$$

We define the Hochschild homology of $A$ as the homology of the Hochschild homological complex associated to $A$, i.e. $H H_{0}(A):=A / \operatorname{Im} d_{1}$ and for $p \in \mathbb{N}^{*}, H H_{p}(A):=\operatorname{Ker} d_{p} / \operatorname{Im} d_{p+1}$.

\section{Presentation of the Koszul complex}

We recall in this section some results about the Koszul complex used below (see the appendix of $[8])$.

\section{$2.1 \quad$ Kontsevich theorem and notations}

As in Section 1.2, we consider $R=\mathbb{C}[\mathbf{z}]$ and $\left(f_{1}, \ldots, f_{m}\right) \in R^{m}$, and we denote by $A$ the quotient $R /\left\langle f_{1}, \ldots, f_{m}\right\rangle$. We assume that we have a complete intersection, i.e. the dimension of the set of solutions of the system $\left\{f_{1}(\mathbf{z})=\cdots=f_{m}(\mathbf{z})=0\right\}$ is $n-m$.

We consider the differential graded algebra

$$
\widetilde{T}=A\left[\eta_{1}, \ldots, \eta_{n} ; b_{1}, \ldots, b_{m}\right]=\frac{\mathbb{C}\left[z_{1}, \ldots, z_{n}\right]}{\left\langle f_{1}, \ldots, f_{m}\right\rangle}\left[\eta_{1}, \ldots, \eta_{n} ; b_{1}, \ldots, b_{m}\right],
$$

\footnotetext{
${ }^{2}$ Two deformations $m=\sum_{j=0}^{p} m_{j} \hbar^{j}, m_{j} \in C^{2}(A)$ and $m^{\prime}=\sum_{j=0}^{p} m_{j}^{\prime} \hbar^{j}, m_{j}^{\prime} \in C^{2}(A)$ are called equivalent if there exists a sequence of linear maps $\varphi_{j}$ from $A$ to $A$ of which the first term $\varphi_{0}$ is the identity of $A$ and such that

$$
\begin{aligned}
& \forall a \in A, \quad \varphi(a)=\sum_{j=0}^{\infty} \varphi_{j}(a) \hbar^{j}, \\
& \forall n \in \mathbb{N}, \quad \sum_{i+j=n} \varphi_{i}\left(m_{j}(a, b)\right)=\sum_{i+j+k=n} m_{i}^{\prime}\left(\varphi_{j}(a), \varphi_{k}(b)\right) .
\end{aligned}
$$
}


where $\eta_{i}:=\frac{\partial}{\partial z_{i}}$ is an odd variable (i.e. the $\eta_{i}$ 's anticommute), and $b_{j}$ an even variable (i.e. the $b_{j}$ 's commute).

$\widetilde{T}$ is endowed with the differential

$$
d_{\widetilde{T}}=\sum_{j=1}^{n} \sum_{i=1}^{m} \frac{\partial f_{i}}{\partial z_{j}} b_{i} \frac{\partial}{\partial \eta_{j}},
$$

and the Hodge grading, defined by $\operatorname{deg}\left(z_{i}\right)=0, \operatorname{deg}\left(\eta_{i}\right)=1, \operatorname{deg}\left(b_{j}\right)=2$.

We may now state the main theorem which will allow us to calculate the Hochschild cohomology:

Theorem 1 (Kontsevich). Under the previous assumptions, the Hochschild cohomology of A is isomorphic to the cohomology of the complex $\left(\widetilde{T}, d_{\widetilde{T}}\right)$ associated with the differential graded algebra $\widetilde{T}$.

Remark 1. Theorem 1 may be seen as a generalization of the Hochschild-Kostant-Rosenberg theorem to the case of non-smooth spaces.

There is no element of negative degree. So the complex is as follows

$$
\widetilde{T}(0) \stackrel{\widetilde{0}}{\longrightarrow} \widetilde{T}(1) \stackrel{d_{\widetilde{T}}^{(1)}}{\longrightarrow} \widetilde{T}(2) \stackrel{d_{\widetilde{T}}^{(2)}}{\longrightarrow} \widetilde{T}(3) \stackrel{d_{\widetilde{T}}^{(3)}}{\longrightarrow} \widetilde{T}(4) \stackrel{d_{\widetilde{T}}^{(4)}}{\longrightarrow} \cdots
$$

For each degree $p$, we choose a basis $\mathcal{B}_{p}$ of $\widetilde{T}(p)$. For example for $p=0, \ldots, 3$, we may take

$$
\begin{aligned}
& \widetilde{T}(0)=A, \\
& \widetilde{T}(1)=A \eta_{1} \oplus \cdots \oplus A \eta_{n}, \\
& \widetilde{T}(2)=A b_{1} \oplus \cdots \oplus A b_{m} \oplus \bigoplus_{i<j} A \eta_{i} \eta_{j}, \\
& \widetilde{T}(3)=\bigoplus_{\substack{i=1, \ldots, m \\
j=1, \ldots, n}} A b_{i} \eta_{j} \oplus \bigoplus_{i<j<k} A \eta_{i} \eta_{j} \eta_{k} .
\end{aligned}
$$

Below we shall make use of the explicit matrices $\operatorname{Mat}_{\mathcal{B}_{p}, \mathcal{B}_{p+1}}\left(d_{\widetilde{T}}^{(p)}\right)$.

Set $H^{0}:=A, H^{1}:=\operatorname{Ker} d_{\widetilde{T}}^{(1)}$ and for $j \geq 2, H^{p}:=\operatorname{Ker} d_{\widetilde{T}}^{(p)} / \operatorname{Im} d_{\widetilde{T}}^{(p-1)}$. According to Theorem 1 , we have, for $p \in \mathbb{N}, H H^{p}(A) \simeq H^{p}$.

There is an analogous of Theorem 1 for the Hochschild homology. We consider the complex

$$
\widetilde{\Omega}=A\left[\xi_{1}, \ldots, \xi_{n} ; a_{1}, \ldots, a_{m}\right],
$$

where $\xi_{i}$ is an odd variable and $a_{j}$ an even variable. $\widetilde{\Omega}$ is endowed with the differential

$$
d_{\widetilde{\Omega}}=\sum_{i=1}^{n} \sum_{j=1}^{m} \frac{\partial f_{j}}{\partial z_{i}} \xi_{i} \frac{\partial}{\partial a_{j}},
$$

and the Hodge grading, defined by $\operatorname{deg}\left(z_{i}\right)=0, \operatorname{deg}\left(\xi_{i}\right)=-1, \operatorname{deg}\left(a_{j}\right)=-2$.

Theorem 2 (Kontsevich). Under the previous assumptions, the Hochschild homology of $A$ is isomorphic to the cohomology of the complex $\left(\widetilde{\Omega}, d_{\widetilde{\Omega}}\right)$

$$
\ldots \stackrel{d_{\tilde{\Omega}}^{(-5)}}{\longrightarrow} \widetilde{\Omega}(-4) \stackrel{d_{\widetilde{\Omega}}^{(-4)}}{\longrightarrow} \widetilde{\Omega}(-3) \stackrel{d_{\widetilde{\Omega}}^{(-3)}}{\longrightarrow} \widetilde{\Omega}(-2) \stackrel{d_{\widetilde{\Omega}}^{(-2)}}{\longrightarrow} \widetilde{\Omega}(-1) \stackrel{d_{\widetilde{\Omega}}^{(-1)}}{\longrightarrow} \widetilde{\Omega}(0)
$$


For each degree $p$, we will choose a basis $\mathcal{V}_{p}$ of $\widetilde{\Omega}(p)$ and we will make use of the explicit matrices $\operatorname{Mat}_{\mathcal{V}_{p}, \mathcal{V}_{p+1}}\left(d_{\widetilde{\Omega}}^{(p)}\right)$. Set $L^{0}:=A / \operatorname{Im} d_{\widetilde{\Omega}}^{(-1)}$, and for $p \geq 1, L^{-p}:=\operatorname{Ker} d_{\widetilde{\Omega}}^{(-p)} / \operatorname{Im} d_{\widetilde{\Omega}}^{(-p-1)}$. According to Theorem 2, we have, for $p \in \mathbb{N}, H H_{p}(A) \simeq L^{-p}$.

For each ideal $J$ of $\mathbb{C}[\mathbf{z}]$, we denote by $J_{A}$ the image of $J$ by the canonical projection

$$
\mathbb{C}[\mathbf{z}] \rightarrow A=\mathbb{C}[\mathbf{z}] /\left\langle f_{1}, \ldots, f_{m}\right\rangle .
$$

Similarly if $\left(g_{1}, \ldots, g_{r}\right) \in A^{r}$ we denote by $\left\langle g_{1}, \ldots, g_{r}\right\rangle_{A}$ the ideal of $A$ generated by $\left(g_{1}, \ldots, g_{r}\right)$. Besides, if $g \in \mathbb{C}[\mathbf{z}]$, and if $J$ is an ideal of $\mathbb{C}[\mathbf{z}]$, we set

$$
\operatorname{Ann}_{J}(g):=\{h \in \mathbb{C}[\mathbf{z}] / h g=0 \bmod J\} .
$$

In particular, $g$ does not divide 0 in $\mathbb{C}[\mathbf{z}] / J$ if and only if $\operatorname{Ann}_{J}(g)=J$. Finally, we denote by $\nabla g$ the gradient of a polynomial $g \in \mathbb{C}[\mathbf{z}]$.

From now on, we consider the case $m=1$ and set $f:=f_{1}$. Moreover, we use the notation $\partial_{j}$ for the partial derivative with respect to $z_{j}$.

\subsection{Particular case where $n=1$ and $m=1$}

In the case where $n=1$ and $m=1$, according to what we have seen, we have for $p \in \mathbb{N}$,

$$
\widetilde{T}(2 p)=A b_{1}^{p}, \quad \widetilde{T}(2 p+1)=A b_{1}^{p} \eta_{1}, \quad \widetilde{\Omega}(-2 p)=A a_{1}^{p}, \quad \widetilde{\Omega}(-2 p-1)=A a_{1}^{p} \xi_{1} .
$$

We deduce

$$
H^{0}=L^{0}=A, \quad H^{1}=\left\{g \eta_{1} / g \in A \text { and } g \partial_{1} f=0\right\},
$$

and for $p \in \mathbb{N}^{*}$,

$$
H^{2 p}=\frac{A b_{1}^{p}}{\left\{g\left(\partial_{1} f\right) b_{1}^{p} / g \in A\right\}}, \quad \text { and } \quad H^{2 p+1}=\left\{g b_{1}^{p} \eta_{1} / g \in A \text { and } g \partial_{1} f=0\right\} .
$$

Similarly, for $p \in \mathbb{N}^{*}$,

$$
L^{-2 p}=\left\{g a_{1}^{p} / g \in A \text { and } g \partial_{1} f=0\right\},
$$

and for $p \in \mathbb{N}$,

$$
L^{-2 p-1}=\frac{A a_{1}^{p} \xi_{1}}{\left\{g\left(\partial_{1} f\right) a_{1}^{p} \xi_{1} / g \in A\right\}} .
$$

Now if $f=z_{1}^{k}$, then

$$
\begin{aligned}
& H^{0}=L^{0}=A=\mathbb{C}\left[z_{1}\right] /\left\langle z_{1}^{k}\right\rangle \simeq \mathbb{C}^{k}, \\
& H^{1}=\left\{g \eta_{1} / g \in A \text { and } k g z_{1}^{k-1}=0\right\} \simeq \mathbb{C}^{k-1}, \quad L^{-1}=\frac{A \xi_{1}}{\left\{g\left(k z_{1}^{k-1}\right) \xi_{1} / g \in A\right\}} \simeq \mathbb{C}^{k-1},
\end{aligned}
$$

and for $p \in \mathbb{N}^{*}$,

$$
H^{2 p} \simeq L^{-2 p-1} \simeq \frac{A b_{1}^{p}}{\left\{g\left(k z_{1}^{k-1}\right) b_{1}^{p} / g \in A\right\}} \simeq \mathbb{C}^{k-1},
$$

and for $p \in \mathbb{N}^{*}$,

$$
H^{2 p+1} \simeq L^{-2 p} \simeq\left\{g b_{1}^{p} \eta_{1} / g \in A \text { and } k g z_{1}^{k-1}=0\right\} \simeq \mathbb{C}^{k-1} .
$$

See [12] for a similar calculation. 


\section{Case $n=2, m=1$. Singular curves of the plane}

\subsection{Singular curves of the plane}

In this section, we recall a result about the weighted homogeneous functions, given in [3, page 181].

Theorem 3 (Classification of weighted homogeneous functions, [3]). The weighted homogeneous functions of two variables and of modality zero reduce, up to equivalence, to the following list of normal forms

\begin{tabular}{|l||c|c|c|c|c|}
\hline Type & $A_{k}$ & $D_{k}$ & $E_{6}$ & $E_{7}$ & $E_{8}$ \\
\hline Normal form & $z_{1}^{k+1}+z_{2}^{2}$ & $z_{1}^{2} z_{2}+z_{2}^{k-1}$ & $z_{1}^{3}+z_{2}^{4}$ & $z_{1}^{3}+z_{1} z_{2}^{3}$ & $z_{1}^{3}+z_{2}^{5}$ \\
\hline
\end{tabular}

The singularities of types $A_{k}, D_{k}, E_{6}, E_{7}, E_{8}$ are called simple singularities. In the two following sections, we will study the Hochschild cohomology of $\mathbb{C}[\mathbf{z}] /\langle f\rangle$, where $f$ is one of the normal forms of the preceding table.

\subsection{Description of the cohomology spaces}

With the help of Theorem 1 we calculate the Hochschild cohomology of $A:=\mathbb{C}\left[z_{1}, z_{2}\right] /\langle f\rangle$, where $f \in \mathbb{C}\left[z_{1}, z_{2}\right]$. We begin by making cochains and differentials explicit, by using the notations of Section 2.1.

The various spaces of the complex are given by

$$
\begin{array}{llrl}
\widetilde{T}(0) & =A, & \widetilde{T}(5)=A b_{1}^{2} \eta_{1} \oplus A b_{1}^{2} \eta_{2}, \\
\widetilde{T}(1)=A \eta_{1} \oplus A \eta_{2}, & & \widetilde{T}(6)=A b_{1}^{3} \oplus A b_{1}^{2} \eta_{1} \eta_{2}, \\
\widetilde{T}(2)=A b_{1} \oplus A \eta_{1} \eta_{2}, & & \widetilde{T}(7)=A b_{1}^{3} \eta_{1} \oplus A b_{1}^{3} \eta_{2}, \\
\widetilde{T}(3)=A b_{1} \eta_{1} \oplus A b_{1} \eta_{2}, & & \widetilde{T}(8)=A b_{1}^{4} \oplus A b_{1}^{3} \eta_{1} \eta_{2}, \\
\widetilde{T}(4)=A b_{1}^{2} \oplus A b_{1} \eta_{1} \eta_{2}, & & \widetilde{T}(9)=A b_{1}^{4} \eta_{1} \oplus A b_{1}^{4} \eta_{2},
\end{array}
$$

i.e., for an arbitrary $p \in \mathbb{N}^{*}$,

$$
\widetilde{T}(2 p)=A b_{1}^{p} \oplus A b_{1}^{p-1} \eta_{1} \eta_{2},
$$

and for an arbitrary $p \in \mathbb{N}$,

$$
\widetilde{T}(2 p+1)=A b_{1}^{p} \eta_{1} \oplus A b_{1}^{p} \eta_{2} .
$$

As in [8], we denote by $\frac{\partial}{\partial \eta_{k}}$ the partial derivative with respect to the variable $\eta_{k}$, for $k \in\{1,2\}$. So, for $\{k, l\}=\{1,2\}$, we have

$$
\frac{\partial}{\partial \eta_{k}}\left(\eta_{k} \wedge \eta_{l}\right)=1 \wedge \eta_{l}=-\eta_{l} \wedge 1
$$

hence

$$
d_{\widetilde{T}}^{(2)}\left(\eta_{k} \eta_{l}\right)=-\frac{\partial f}{\partial z_{k}} b_{1} \eta_{l}+\frac{\partial f}{\partial z_{l}} b_{1} \eta_{k}
$$

The matrices of $d_{\widetilde{T}}$ are therefore given by

$$
\operatorname{Mat}_{\mathcal{B}_{2 p}, \mathcal{B}_{2 p+1}}\left(d_{\widetilde{T}}^{(2 p)}\right)=\left(\begin{array}{cc}
0 & \partial_{2} f \\
0 & -\partial_{1} f
\end{array}\right)
$$




$$
\operatorname{Mat}_{\mathcal{B}_{2 p+1}, \mathcal{B}_{2 p+2}}\left(d_{\widetilde{T}}^{(2 p+1)}\right)=\left(\begin{array}{cc}
\partial_{1} f & \partial_{2} f \\
0 & 0
\end{array}\right)
$$

We deduce a simpler expression for the cohomology spaces

$$
\begin{aligned}
H^{0} & =A, \\
H^{1} & =\left\{g_{1} \eta_{1}+g_{2} \eta_{2} /\left(g_{1}, g_{2}\right) \in A^{2} \text { and } g_{1} \partial_{1} f+g_{2} \partial_{2} f=0\right\} \\
& \simeq\left\{\mathbf{g}=\left(\begin{array}{c}
g_{1} \\
g_{2}
\end{array}\right) \in A^{2} / \mathbf{g} \cdot \nabla f=0\right\} .
\end{aligned}
$$

For $p \in \mathbb{N}^{*}$,

$$
\begin{aligned}
& H^{2 p}=\frac{\left\{g_{1} b_{1}^{p}+g_{2} b_{1}^{p-1} \eta_{1} \eta_{2} /\left(g_{1}, g_{2}\right) \in A^{2} \text { and } g_{2} \partial_{1} f=g_{2} \partial_{2} f=0\right\}}{\left\{\left(g_{1} \partial_{1} f+g_{2} \partial_{2} f\right) b_{1}^{p} /\left(g_{1}, g_{2}\right) \in A^{2}\right\}} \\
& \simeq \frac{\left\{\mathbf{g}=\left(\begin{array}{c}
g_{1} \\
g_{2}
\end{array}\right) \in A^{2} / g_{2} \partial_{1} f=g_{2} \partial_{2} f=0\right\}}{\left\{\left(\begin{array}{c}
\mathbf{g} \cdot \nabla f \\
0
\end{array}\right) / \mathbf{g} \in A^{2}\right\}} \\
& \simeq \frac{A}{\left\langle\partial_{1} f, \partial_{2} f\right\rangle_{A}} \oplus\left\{g \in A / g \partial_{1} f=g \partial_{2} f=0\right\}, \\
& H^{2 p+1}=\frac{\left\{g_{1} b_{1}^{p} \eta_{1}+g_{2} b_{1}^{p} \eta_{2} /\left(g_{1}, g_{2}\right) \in A^{2} \text { and } g_{1} \partial_{1} f+g_{2} \partial_{2} f=0\right\}}{\left\{g_{2}\left(\partial_{2} f b_{1}^{p} \eta_{1}-\partial_{1} f b_{1}^{p} \eta_{2}\right) / g_{2} \in A\right\}} \\
& \simeq \frac{\left\{\mathbf{g}=\left(\begin{array}{c}
g_{1} \\
g_{2}
\end{array}\right) \in A^{2} / \mathbf{g} \cdot \nabla f=0\right\}}{\left\{g_{2}\left(\begin{array}{c}
\partial_{2} f \\
-\partial_{1} f
\end{array}\right) / g_{2} \in A\right\}} \text {. }
\end{aligned}
$$

Remark 2. We recover a result of [8] (here, we use the notations of [8]). According to Theorem 3.8 of [8], we have $\operatorname{Hoch}_{2 p}=\operatorname{Hoch}_{2 p, p} \oplus \operatorname{Hoch}_{2 p, p+1}$ and $\operatorname{Hoch}_{2 p+1}=\operatorname{Hoch}_{2 p+1, p+1}$, so $\operatorname{Hoch}_{2 p, k}=0$ if $k \notin\{p, p+1\}$, and $H_{o c h}{ }_{2 p+1, k}=0$ if $k \neq p+1$. By using Section 4.1 of [8], we deduce $H^{2 p, k}=0$ if $k \notin\{p, p+1\}$, and $H^{2 p+1, k}=0$ if $k \neq p+1$. Hence $H^{2 p}=H^{2 p, p} \oplus H^{2 p, p+1}$ and $H^{2 p+1}=H^{2 p+1, p+1}$. So Theorem 4.9 of [8] gives the cohomology spaces which we have just obtained.

It remains to determine these spaces more explicitly. This will be done in the two following sections.

\subsection{Explicit calculations in the particular case where $f$ has separate variables}

In this section, we consider the polynomial $f=a_{1} z_{1}^{k}+a_{2} z_{2}^{l}$, with $k \geq 2, l \geq 2$, and $\left(a_{1}, a_{2}\right) \in\left(\mathbb{C}^{*}\right)^{2}$. The partial derivatives of $f$ are $\partial_{1} f=k a_{1} z_{1}^{k-1}$ and $\partial_{2} f=l a_{2} z_{2}^{l-1}$.

We already have

$$
H^{0}=\mathbb{C}\left[z_{1}, z_{2}\right] /\left\langle a_{1} z_{1}^{k}+a_{2} z_{2}^{l}\right\rangle .
$$

Besides, as $f$ is weighted homogeneous, Euler's formula gives $\frac{1}{k} z_{1} \partial_{1} f+\frac{1}{l} z_{2} \partial_{2} f=f$. So we have the inclusion $\langle f\rangle \subset\left\langle\partial_{1} f, \partial_{2} f\right\rangle$, hence

$$
\frac{A}{\left\langle\partial_{1} f, \partial_{2} f\right\rangle_{A}} \simeq \frac{\mathbb{C}\left[z_{1}, z_{2}\right]}{\left\langle\partial_{1} f, \partial_{2} f\right\rangle} \simeq \operatorname{Vect}\left(z_{1}^{i} z_{2}^{j} / i \in \llbracket 0, k-2 \rrbracket, j \in \llbracket 0, l-2 \rrbracket\right) .
$$


But $\partial_{1} f$ and $f$ are relatively prime, just as $\partial_{2} f$ and $f$ are, hence if $g \in A$ satisfies $g \partial_{1} f=0$ $\bmod \langle f\rangle$, then $g \in\langle f\rangle$, i.e. $g$ is zero in $A$. So,

$$
H^{2 p} \simeq \operatorname{Vect}\left(z_{1}^{i} z_{2}^{j} / i \in \llbracket 0, k-2 \rrbracket, j \in \llbracket 0, l-2 \rrbracket\right) \simeq \mathbb{C}^{(k-1)(l-1)} .
$$

We now determine the set

$$
\left\{\mathbf{g}=\left(\begin{array}{l}
g_{1} \\
g_{2}
\end{array}\right) \in A^{2} / \mathbf{g} \cdot \nabla f=0\right\} .
$$

First we have

$$
\left\langle f, \partial_{1} f\right\rangle=\left\langle a_{1} z_{1}^{k}+a_{2} z_{2}^{l}, z_{1}^{k-1}\right\rangle=\left\langle z_{2}^{l}, z_{1}^{k-1}\right\rangle .
$$

So the only monomials which are not in this ideal are the elements $z_{1}^{i} z_{2}^{j}$ with $i \in \llbracket 0, k-2 \rrbracket$ and $j \in \llbracket 0, l-1 \rrbracket$. Every polynomial $P \in \mathbb{C}[\mathbf{z}]$ may be written in the form

$$
P=\alpha f+\beta \partial_{1} f+\sum_{\substack{i=0, \ldots, k-2 \\ j=0, \ldots, l-1}} a_{i j} z_{1}^{i} z_{2}^{j}
$$

with $\alpha, \beta \in \mathbb{C}[\mathbf{z}]$ and $a_{i j} \in \mathbb{C}$. Therefore, the polynomials $P \in \mathbb{C}[\mathbf{z}]$ such that $P \partial_{2} f \in\left\langle f, \partial_{1} f\right\rangle$ are the elements

$$
P=\alpha f+\beta \partial_{1} f+\sum_{\substack{i=0, \ldots, k-2 \\ j=1, \ldots, l-1}} a_{i j} z_{1}^{i} z_{2}^{j}
$$

So we have calculated $\operatorname{Ann}_{\left\langle f, \partial_{1} f\right\rangle}\left(\partial_{2} f\right)$. Let $\mathbf{g}=\left(\begin{array}{c}g_{1} \\ g_{2}\end{array}\right) \in A^{2}$ satisfy the equation

$$
\mathbf{g} \cdot \nabla f=0 \bmod \langle f\rangle .
$$

Then we have

$$
g_{2} \partial_{2} f=0 \bmod \left\langle f, \partial_{1} f\right\rangle,
$$

i.e. $g_{2} \in \operatorname{Ann}_{\left\langle f, \partial_{1} f\right\rangle}\left(\partial_{2} f\right)$, i.e. again

$$
g_{2}=\alpha f+\beta \partial_{1} f+\sum_{\substack{i=0, \ldots, k-2 \\ j=1, \ldots, l-1}} a_{i j} z_{1}^{i} z_{2}^{j}, \quad \text { with }(\alpha, \beta) \in \mathbb{C}[\mathbf{z}]^{2} .
$$

It follows that

$$
g_{1} \partial_{1} f+\alpha f \partial_{2} f+\beta \partial_{1} f \partial_{2} f+\sum_{\substack{i=0, \ldots, k-2 \\ j=1, \ldots, l-1}} a_{i j} z_{1}^{i} z_{2}^{j} \partial_{2} f \in\langle f\rangle .
$$

From the equality $z_{2} \partial_{2} f=l f-\frac{l}{k} z_{1} \partial_{1} f$, one deduces

$$
\partial_{1} f\left(g_{1}+\beta \partial_{2} f-\frac{l}{k} \sum_{\substack{i=0, \ldots, k-2 \\ j=1, \ldots, l-1}} a_{i j} z_{1}^{i+1} z_{2}^{j-1}\right) \in\langle f\rangle,
$$


i.e.

$$
g_{1}=-\beta \partial_{2} f+\frac{l}{k} \sum_{\substack{i=0, \ldots, k-2 \\ j=1, \ldots, l-1}} a_{i j} z_{1}^{i+1} z_{2}^{j-1}+\delta f, \quad \text { with } \delta \in \mathbb{C}[\mathbf{z}]
$$

Then we verify that the elements $g_{1}$ and $g_{2}$ obtained in this way are indeed solutions of equation (2).

Finally, we have

$$
\begin{aligned}
&\left\{\mathbf{g} \in A^{2} / \mathbf{g} \cdot \nabla f=0\right\} \\
& \quad=\left\{-\beta\left(\begin{array}{c}
\partial_{2} f \\
-\partial_{1} f
\end{array}\right)+\sum_{\substack{i=0, \ldots, k-2 \\
j=1, \ldots, l-1}} a_{i j} z_{1}^{i} z_{2}^{j-1}\left(\begin{array}{c}
\frac{l}{k} z_{1} \\
z_{2}
\end{array}\right) / \beta \in A \text { and } a_{i j} \in \mathbb{C}\right\} .
\end{aligned}
$$

We immediately deduce the cohomology spaces of odd degree:

$$
\begin{aligned}
& \forall p \geq 1, \quad H^{2 p+1} \simeq \mathbb{C}^{(k-1)(l-1)} \\
& H^{1} \simeq \mathbb{C}^{(k-1)(l-1)} \oplus \mathbb{C}\left[z_{1}, z_{2}\right] /\left\langle a_{1} z_{1}^{k}+a_{2} z_{2}^{l}\right\rangle,
\end{aligned}
$$

where the direct sum results from the following argument: if we have

$$
-\beta\left(\begin{array}{c}
\partial_{2} f \\
-\partial_{1} f
\end{array}\right)=\sum_{\substack{i=0, \ldots, k-2 \\
j=1, \ldots, l-1}} a_{i j} z_{1}^{i} z_{2}^{j-1}\left(\begin{array}{c}
\frac{l}{k} z_{1} \\
z_{2}
\end{array}\right) \bmod \langle f\rangle,
$$

then

$$
v:=-\beta l a_{2} z_{2}^{l-1}-\sum_{\substack{i=0, \ldots, k-2 \\ j=1, \ldots, l-1}} \frac{l}{k} a_{i j} z_{1}^{i+1} z_{2}^{j-1} \in\langle f\rangle .
$$

And by a Euclidian division in $\left(\mathbb{C}\left[z_{2}\right]\right)\left[z_{1}\right]$, we may write $\beta=f q+r$, where the $z_{1}$-degree of $r$ is smaller or equal to $k-1$. So the $z_{1}$-degree of $v$ is also smaller or equal to $k-1$, thus $v \in\langle f\rangle$ implies $\beta=0$ and $a_{i j}=0$.

Remark 3. We obtain in particular the cohomology for the cases where $f=z_{1}^{k+1}+z_{2}^{2}\left(k \in \mathbb{N}^{*}\right)$, $f=z_{1}^{3}+z_{2}^{4}$ and $f=z_{1}^{3}+z_{2}^{5}$. These cases correspond respectively to the weighted homogeneous functions of types $A_{k}, E_{6}$ and $E_{8}$ given in Theorem 3.

The table below summarizes the results we have just obtained for the three particular cases

\begin{tabular}{|l||l|l|l|}
\hline & $H^{0}$ & $H^{1}$ & $H^{p}, p \geq 2$ \\
\hline \hline$A_{k}$ & $\mathbb{C}[\mathbf{z}] /\left\langle z_{1}^{k+1}+z_{2}^{2}\right\rangle$ & $\mathbb{C}[\mathbf{z}] /\left\langle z_{1}^{k+1}+z_{2}^{2}\right\rangle \oplus \mathbb{C}^{k}$ & $\mathbb{C}^{k}$ \\
\hline$E_{6}$ & $\mathbb{C}[\mathbf{z}] /\left\langle z_{1}^{3}+z_{2}^{4}\right\rangle$ & $\mathbb{C}[\mathbf{z}] /\left\langle z_{1}^{3}+z_{2}^{4}\right\rangle \oplus \mathbb{C}^{6}$ & $\mathbb{C}^{6}$ \\
\hline$E_{8}$ & $\mathbb{C}[\mathbf{z}] /\left\langle z_{1}^{3}+z_{2}^{5}\right\rangle$ & $\mathbb{C}[\mathbf{z}] /\left\langle z_{1}^{3}+z_{2}^{5}\right\rangle \oplus \mathbb{C}^{8}$ & $\mathbb{C}^{8}$ \\
\hline
\end{tabular}

The cases where $f=z_{1}^{2} z_{2}+z_{2}^{k-1}$ and $f=z_{1}^{3}+z_{1} z_{2}^{3}$, i.e. respectively $D_{k}$ and $E_{7}$, will be studied in the next section. 


\subsection{Explicit calculations for $D_{k}$ and $E_{7}$}

To study these particular cases, we use the following result about Groebner bases (Theorem 4). First, recall the definition of a Groebner basis. For $g \in \mathbb{C}[\mathbf{z}]$, we denote by $\operatorname{lt}(g)$ its leading term (for the lexicographic order). Given a non-trivial ideal $J$ of $\mathbb{C}[\mathbf{z}]$, a Groebner basis of $J$ is a finite subset $G_{J}$ of $J \backslash\{0\}$ such that for all $f \in J \backslash\{0\}$, there exists $g \in G_{J}$ such that $\operatorname{lt}(g)$ divides $\operatorname{lt}(f)$. See $[15]$ for more details.

Definition 1. Let $J$ be a non-trivial ideal of $\mathbb{C}[\mathbf{z}]$ and let $G_{J}:=\left[g_{1}, \ldots, g_{r}\right]$ be a Groebner basis of $J$. We call set of the $G_{J}$-standard terms, the set of all monomials of $\mathbb{C}[\mathbf{z}]$ that are not divisible by any of $\operatorname{lt}\left(g_{1}\right), \ldots, \operatorname{lt}\left(g_{r}\right)$.

Theorem 4 (Macaulay). The set of the $G_{J}$-standard terms forms a basis of the quotient vector space $\mathbb{C}[\mathbf{z}] / J$.

\subsubsection{Case of $f=z_{1}^{2} z_{2}+z_{2}^{k-1}$, i.e. $D_{k}$}

Here we have

$$
f=z_{1}^{2} z_{2}+z_{2}^{k-1}, \quad \partial_{1} f=2 z_{1} z_{2} \quad \text { and } \quad \partial_{2} f=z_{1}^{2}+(k-1) z_{2}^{k-2} .
$$

A Groebner basis of the ideal $\left\langle f, \partial_{2} f\right\rangle$ is

$$
B:=\left[b_{1}, b_{2}\right]=\left[z_{1}^{2}+(k-1) z_{2}^{k-2}, z_{2}^{k-1}\right] .
$$

So the set of the standard terms is

$$
\left\{z_{1}^{i} z_{2}^{j} / i \in\{0,1\} \text { and } j \in \llbracket 0, k-2 \rrbracket\right\} .
$$

We may now solve the equation $p \partial_{1} f=0$ in $\mathbb{C}[\mathbf{z}] /\left\langle f, \partial_{2} f\right\rangle$. In fact, by writing

$$
p:=\sum_{\substack{i=0,1 \\ j=0, \ldots, k-2}} a_{i j} z_{1}^{i} z_{2}^{j},
$$

the equation becomes

$$
q:=\sum_{\substack{i=0,1 \\ j=0, \ldots, k-2}} a_{i j} z_{1}^{i+1} z_{2}^{j+1} \in\left\langle f, \partial_{2} f\right\rangle .
$$

We look for the normal form of the element $q$ modulo the ideal $\left\langle f, \partial_{2} f\right\rangle$.

The multivariate division of $q$ by $B$ is $q=q_{1} b_{1}+q_{2} b_{2}+r$ with $r=\sum_{j=0}^{k-3} a_{0, j} z_{1} z_{2}^{j+1}$. Thus the solution in $\mathbb{C}[\mathbf{z}] /\left\langle f, \partial_{2} f\right\rangle$ is

$$
p=a_{0, k-2} z_{2}^{k-2}+\sum_{j=0}^{k-2} a_{1, j} z_{1} z_{2}^{j} .
$$

But the equation

$$
\mathbf{g} \cdot \nabla f=0 \quad \bmod \langle f\rangle
$$

yields

$$
g_{1} \partial_{1} f=0 \bmod \left\langle f, \partial_{2} f\right\rangle,
$$


i.e.

$$
g_{1}=\alpha f+\beta \partial_{2} f+a z_{2}^{k-2}+\sum_{j=0}^{k-2} b_{j} z_{1} z_{2}^{j}, \quad \text { with }(\alpha, \beta) \in \mathbb{C}[\mathbf{z}]^{2} \text { and } a, b_{j} \in \mathbb{C} .
$$

Hence

$$
g_{2} \partial_{2} f+\beta \partial_{1} f \partial_{2} f+a z_{2}^{k-2} \partial_{1} f+\sum_{j=0}^{k-2} b_{j} z_{1} z_{2}^{j} \partial_{1} f \in\langle f\rangle .
$$

And with the equalities,

$$
z_{2}^{k-1}=\frac{1}{2-k}\left(f-z_{2} \partial_{2} f\right)=-\frac{1}{2-k} z_{2} \partial_{2} f \bmod \langle f\rangle,
$$

and

$$
\frac{k-2}{2} z_{1} \partial_{1} f+z_{2} \partial_{2} f=(k-1) f \quad(\text { Euler }),
$$

we obtain

$$
\partial_{2} f\left(g_{2}+\beta \partial_{1} f-\frac{2 a}{2-k} z_{1} z_{2}+\sum_{j=0}^{k-2} b_{j} \frac{2}{2-k} z_{2}^{j+1}\right) \in\langle f\rangle .
$$

i.e.,

$$
g_{2}=-\beta \partial_{1} f+\frac{2 a}{2-k} z_{1} z_{2}-\sum_{j=0}^{k-2} b_{j} \frac{2}{2-k} z_{2}^{j+1}+\delta f, \quad \text { with } \delta \in \mathbb{C}[\mathbf{z}] .
$$

So

$$
\begin{aligned}
& \left\{\mathbf{g} \in A^{2} / \mathbf{g} \cdot \nabla f=0\right\} \\
& =\left\{\beta\left(\begin{array}{c}
\partial_{2} f \\
-\partial_{1} f
\end{array}\right)+a\left(\begin{array}{c}
z_{2}^{k-2} \\
\frac{2}{2-k} z_{1} z_{2}
\end{array}\right)+\sum_{j=0}^{k-2} b_{j} z_{2}^{j}\left(\begin{array}{c}
z_{1} \\
-\frac{2}{2-k} z_{2}
\end{array}\right) / \beta \in A, a, b_{j} \in \mathbb{C}\right\} .
\end{aligned}
$$

On the other hand, a Groebner basis of $\left\langle\partial_{1} f, \partial_{2} f\right\rangle$ is $\left[z_{1}^{2}+(k-1) z_{2}^{k-2}, z_{1} z_{2}, z_{2}^{k-1}\right]$, thus

$$
\mathbb{C}[\mathbf{z}] /\left\langle\partial_{1} f, \partial_{2} f\right\rangle \simeq \operatorname{Vect}\left(z_{1}, 1, z_{2}, \ldots, z_{2}^{k-2}\right) .
$$

Let us summarize (by using, for the direct sum, the same argument as in Section 3.2):

$$
\begin{aligned}
& H^{0}=\mathbb{C}[\mathbf{z}] /\left\langle z_{1}^{2} z_{2}+z_{2}^{k-1}\right\rangle, \\
& H^{1} \simeq \mathbb{C}[\mathbf{z}] /\left\langle z_{1}^{2} z_{2}+z_{2}^{k-1}\right\rangle \oplus \mathbb{C}^{k}, \\
& H^{2 p} \simeq \mathbb{C}^{k} \\
& H^{2 p+1} \simeq \mathbb{C}^{k}
\end{aligned}
$$

\subsubsection{Case of $f=z_{1}^{3}+z_{1} z_{2}^{3}$, i.e. $E_{7}$}

Here we have $\partial_{1} f=3 z_{1}^{2}+z_{2}^{3}$ and $\partial_{2} f=3 z_{1} z_{2}^{2}$. A Groebner basis of the ideal $\left\langle f, \partial_{1} f\right\rangle$ is $\left[3 z_{1}^{2}+z_{2}^{3}, z_{1} z_{2}^{3}, z_{2}^{6}\right]$, and a Groebner basis of $\left\langle\partial_{1} f, \partial_{2} f\right\rangle$ is $\left[3 z_{1}^{2}+z_{2}^{3}, z_{1} z_{2}^{2}, z_{2}^{5}\right]$. By an analogous proof, we obtain

$$
\begin{aligned}
& H^{0}=\mathbb{C}[\mathbf{z}] /\left\langle z_{1}^{3}+z_{1} z_{2}^{3}\right\rangle, \\
& H^{1} \simeq \mathbb{C}[\mathbf{z}] /\left\langle z_{1}^{3}+z_{1} z_{2}^{3}\right\rangle \oplus \mathbb{C}^{7}, \\
& H^{2 p} \simeq \mathbb{C}^{7}, \\
& H^{2 p+1} \simeq \mathbb{C}^{7} .
\end{aligned}
$$




\subsection{Homology}

The study is the same as the one of the Hochschild cohomology: to get the Hochschild homology is equivalent to compute the cohomology of the complex $\left(\widetilde{\Omega}, d_{\widetilde{\Omega}}\right)$ described in Section 2.1. We have $\widetilde{\Omega}(0)=A, \widetilde{\Omega}(-2 p)=A a_{1}^{p} \oplus A a_{1}^{p-1} \xi_{1} \xi_{2}$ for $p \in \mathbb{N}^{*}$, and $\widetilde{\Omega}(-2 p-1)=A a_{1}^{p} \xi_{1} \oplus A a_{1}^{p} \xi_{2}$ for $p \in \mathbb{N}$. This defines the bases $\mathcal{V}_{p}$. The differential is $d_{\widetilde{\Omega}}=\left(\xi_{1} \partial_{1} f+\xi_{2} \partial_{2} f\right) \frac{\partial}{\partial a_{1}}$.

So we obtain, for $p \in \mathbb{N}^{*}$, the matrices

$$
\operatorname{Mat}_{-2 p}, \mathcal{V}_{-2 p+1}\left(d_{\widetilde{\Omega}}^{(-2 p)}\right)=\left(\begin{array}{ll}
p \partial_{1} f & 0 \\
p \partial_{2} f & 0
\end{array}\right)
$$

and

$$
\operatorname{Mat}_{\mathcal{V}_{-2 p-1}, \mathcal{V}_{-2 p}}\left(d_{\widetilde{\Omega}}^{(-2 p-1)}\right)=\left(\begin{array}{cc}
0 & 0 \\
-p \partial_{2} f & p \partial_{1} f
\end{array}\right) .
$$

The cohomology spaces read as

$$
L^{0}=A, \quad L^{-1}=\frac{A^{2}}{\{g \nabla f / g \in A\}} .
$$

For $p \in \mathbb{N}^{*}$,

$$
\begin{gathered}
L^{-2 p} \simeq \frac{\left\{\left(\begin{array}{c}
g_{1} \\
g_{2}
\end{array}\right) \in A^{2} / p g_{1} \partial_{1} f=p g_{1} \partial_{2} f=0\right\}}{\left\{\left(\begin{array}{c}
-(p+1) g_{1} \partial_{2} f+(p+1) g_{2} \partial_{1} f
\end{array}\right) /\left(\begin{array}{c}
g_{1} \\
g_{2}
\end{array}\right) \in A^{2}\right\}} \\
\simeq\left\{g \in A / g \partial_{1} f=g \partial_{2} f=0\right\} \oplus \frac{A}{\langle\nabla f\rangle_{A}} .
\end{gathered}
$$

For $p \in \mathbb{N}^{*}$,

$$
L^{-2 p-1} \simeq \frac{\left\{\left(\begin{array}{l}
g_{1} \\
g_{2}
\end{array}\right) \in A^{2} /-p g_{1} \partial_{2} f+p g_{2} \partial_{1} f=0\right\}}{\left\{\left(\begin{array}{c}
(p+1) g_{1} \partial_{1} f \\
(p+1) g_{1} \partial_{2} f
\end{array}\right) /\left(\begin{array}{c}
g_{1} \\
g_{2}
\end{array}\right) \in A^{2}\right\}} \simeq \frac{\left\{\mathbf{g} \in A^{2} / \operatorname{det}(\nabla f, \mathbf{g})=0\right\}}{\{g \nabla f / g \in A\}} .
$$

From now on, we assume that $f$ has separate variables, or $f$ is of type $D_{k}$ or $E_{7}$. Then we have $\left\{g \in A / g \partial_{1} f=g \partial_{2} f=0\right\}=\{0\}$, and according to Euler's formula, for $p \in \mathbb{N}, L^{-2 p} \simeq$ $\frac{A}{\langle\nabla f\rangle_{A}} \simeq \frac{\mathbb{C}[\mathbf{z}]}{\langle\nabla f\rangle}$. For the computation of $\left\{\mathbf{g} \in A^{2} / \operatorname{det}(\nabla f, \mathbf{g})=0\right\}$ and $\frac{A^{2}}{\{g \nabla f / g \in A\}}$, we proceed with Groebner bases as in Section 3.3. For example, we do it for $f=z_{1}^{2} z_{2}+z_{2}^{k-1}$ (i.e. type $D_{k}$ ).

Let $\mathbf{g} \in A^{2}$ be such that $\operatorname{det}(\nabla f, \mathbf{g})=0$. Then $g_{2} \partial_{1} f=0 \bmod \left\langle f, \partial_{2} f\right\rangle$, i.e., according to Section 3.4.1,

$$
g_{2}=\alpha f+\beta \partial_{2} f+a z_{2}^{k-2}+\sum_{j=0}^{k-2} b_{j} z_{1} z_{2}^{j},
$$

with $(\alpha, \beta) \in \mathbb{C}[\mathbf{z}]^{2}$ and $a, b_{j} \in \mathbb{C}$. Hence

$$
-g_{1} \partial_{2} f+\alpha f \partial_{1} f+\beta \partial_{2} f \partial_{1} f+a z_{2}^{k-2} \partial_{1} f+\sum_{j=0}^{k-2} b_{j} z_{1} z_{2}^{j} \partial_{1} f \in\langle f\rangle .
$$


With the equalities,

$$
z_{2}^{k-1}=\frac{1}{2-k}\left(f-z_{2} \partial_{2} f\right)=-\frac{1}{2-k} z_{2} \partial_{2} f \bmod \langle f\rangle,
$$

and

$$
\frac{k-2}{2} z_{1} \partial_{1} f+z_{2} \partial_{2} f=(k-1) f \quad(\text { Euler })
$$

we obtain

$$
\partial_{2} f\left(-g_{1}+\beta \partial_{1} f-\frac{2 a}{2-k} z_{1} z_{2}+\sum_{j=0}^{k-2} b_{j} \frac{2}{2-k} z_{2}^{j+1}\right) \in\langle f\rangle .
$$

i.e.,

$$
g_{1}=\beta \partial_{1} f-\frac{2 a}{2-k} z_{1} z_{2}+\sum_{j=0}^{k-2} b_{j} \frac{2}{2-k} z_{2}^{j+1}+\delta f, \quad \text { with } \delta \in \mathbb{C}[\mathbf{z}]
$$

So

$$
\begin{aligned}
\{\mathbf{g} \in & \left.A^{2} / \operatorname{det}(\nabla f, \mathbf{g})=0\right\} \\
& =\left\{\beta \nabla f+a\left(\begin{array}{c}
-\frac{2}{2-k} z_{1} z_{2} \\
z_{2}^{k-2}
\end{array}\right)+\sum_{j=0}^{k-2} b_{j} z_{2}^{j}\left(\begin{array}{c}
\frac{2}{2-k} z_{2} \\
z_{1}
\end{array}\right) / \beta \in A, a, b_{j} \in \mathbb{C}\right\} .
\end{aligned}
$$

We have $\{g \nabla f / g \in A\} \subset\left\{\mathbf{g} \in A^{2} / \operatorname{det}(\nabla f, \mathbf{g})=0\right\}$, thus

$$
\operatorname{dim}\left(A^{2} /\{g \nabla f / g \in A\}\right) \geq \operatorname{dim}\left(A^{2} /\left\{\mathbf{g} \in A^{2} / \operatorname{det}(\nabla f, \mathbf{g})=0\right\}\right) .
$$

Since $A^{2} /\left\{\mathbf{g} \in A^{2} / \operatorname{det}(\nabla f, \mathbf{g})=0\right\} \simeq\left\{\operatorname{det}(\nabla f, \mathbf{g}) / \mathbf{g} \in A^{2}\right\}$, and since the map

$$
g \in A \mapsto \operatorname{det}\left(\nabla f,\left(\begin{array}{l}
g \\
0
\end{array}\right)\right) \in\left\{\operatorname{det}(\nabla f, \mathbf{g}) / \mathbf{g} \in A^{2}\right\}
$$

is injective, we deduce that $A^{2} /\{g \nabla f / g \in A\}$ is infinite-dimensional.

We collect in the following table the results for the Hochschild homology in the various cases

\begin{tabular}{|l||l|l|l|}
\hline Type & $H H_{0}=A$ & $H H_{1}$ & $H H_{p}, p \geq 2$ \\
\hline \hline$A_{k}$ & $\mathbb{C}[\mathbf{z}] /\left\langle z_{1}^{k+1}+z_{2}^{2}\right\rangle$ & $A^{2} / A \nabla f$ & $\mathbb{C}^{k}$ \\
\hline$D_{k}$ & $\mathbb{C}[\mathbf{z}] /\left\langle z_{1}^{2} z_{2}+z_{2}^{k-1}\right\rangle$ & $A^{2} / A \nabla f$ & $\mathbb{C}^{k}$ \\
\hline$E_{6}$ & $\mathbb{C}[\mathbf{z}] /\left\langle z_{1}^{3}+z_{2}^{4}\right\rangle$ & $A^{2} / A \nabla f$ & $\mathbb{C}^{6}$ \\
\hline$E_{7}$ & $\mathbb{C}[\mathbf{z}] /\left\langle z_{1}^{3}+z_{1} z_{2}^{3}\right\rangle$ & $A^{2} / A \nabla f$ & $\mathbb{C}^{7}$ \\
\hline$E_{8}$ & $\mathbb{C}[\mathbf{z}] /\left\langle z_{1}^{3}+z_{2}^{5}\right\rangle$ & $A^{2} / A \nabla f$ & $\mathbb{C}^{8}$ \\
\hline
\end{tabular}

\section{Case $n=3, m=1$. Klein surfaces}

\subsection{Klein surfaces}

Given a finite group $G$ acting on $\mathbb{C}^{n}$, we associate to it, according to Erlangen program of Klein, the quotient space $\mathbb{C}^{n} / G$, i.e. the space whose points are the orbits under the action 
of $G$; it is an algebraic variety, and the polynomial functions on this variety are the polynomial functions on $\mathbb{C}^{n}$ which are $G$-invariant. In the case of $\mathbf{S L}_{2} \mathbb{C}$, invariant theory allows us to associate a polynomial to any finite subgroup, as explained in Proposition 4. Thus, to every finite subgroup of $\mathbf{S L}_{2} \mathbb{C}$ is associated the zero set of this polynomial; it is an algebraic variety, called a Klein surface.

In this section we recall some results about these surfaces. See the references [17] and [7] for more details.

Proposition 3. Every finite subgroup of $\mathbf{S L}_{2} \mathbb{C}$ is conjugate to one of the following groups:

- $A_{k}$ (cyclic), $k \geq 1,\left|A_{k}\right|=k$;

- $D_{k}$ (dihedral), $k \geq 1,\left|D_{k}\right|=4 k$;

- $E_{6}$ (tetrahedral), $\left|E_{6}\right|=24$;

- $E_{7}$ (octahedral), $\left|E_{7}\right|=48$;

- $E_{8}$ (icosahedral), $\left|E_{8}\right|=120$.

Proposition 4. Let $G$ be one of the groups of the preceding list. The ring of invariants is the following

$$
\mathbb{C}[x, y]^{G}=\mathbb{C}\left[e_{1}, e_{2}, e_{3}\right]=\mathbb{C}\left[e_{1}, e_{2}\right] \oplus e_{3} \mathbb{C}\left[e_{1}, e_{2}\right] \simeq \mathbb{C}\left[z_{1}, z_{2}, z_{3}\right] /\langle f\rangle,
$$

where the invariants $e_{j}$ are homogeneous polynomials, with $e_{1}$ and $e_{2}$ algebraically independent, and where $f$ is a weighted homogeneous polynomial with an isolated singularity at the origin. These polynomials are given in the following table.

We call Klein surface the algebraic hyper-surface defined by $\left\{\mathbf{z} \in \mathbb{C}^{3} / f(\mathbf{z})=0\right\}$.

\begin{tabular}{|c|c|c|c|}
\hline$G$ & $e_{1}, e_{2}, e_{3}$ & $f$ & $\mathbb{C}\left[z_{1}, z_{2}, z_{3}\right] /\left\langle\partial_{1} f, \partial_{2} f, \partial_{3} f\right\rangle$ \\
\hline$A_{k}$ & $\begin{array}{l}e_{1}=x^{k} \\
e_{2}=y^{k} \\
e_{3}=x y\end{array}$ & $-k\left(z_{1} z_{2}-z_{3}^{k}\right)$ & $\begin{array}{l}\operatorname{Vect}\left(1, z_{3}, \ldots, z_{3}^{k-2}\right) \\
\operatorname{dim}=k-1\end{array}$ \\
\hline$D_{k}$ & $\begin{array}{l}e_{1}=x^{2 k+1} y+(-1)^{k+1} x y^{2 k+1} \\
e_{2}=x^{2 k}+(-1)^{k} y^{2 k} \\
e_{3}=x^{2} y^{2}\end{array}$ & $\begin{array}{l}\lambda_{k}\left((-1)^{k} z_{1}^{2}+(-1)^{k+1} z_{2}^{2} z_{3}+4 z_{3}^{k+1}\right) \\
\text { with } \lambda_{k}=2 k(-1)^{k+1}\end{array}$ & $\begin{array}{l}\operatorname{Vect}\left(1, z_{2}, z_{3}, \ldots, z_{3}^{k-1}\right) \\
\operatorname{dim}=k+1\end{array}$ \\
\hline$E_{6}$ & $\begin{array}{l}e_{1}=33 y^{8} x^{4}-y^{12}+33 y^{4} x^{8}-x^{12} \\
e_{2}=14 y^{4} x^{4}+x^{8}+y^{8} \\
e_{3}=x^{5} y-x y^{5}\end{array}$ & $4\left(z_{1}^{2}-z_{2}^{3}+108 z_{3}^{4}\right)$ & $\begin{array}{l}\operatorname{Vect}\left(1, z_{2}, z_{3}, z_{2} z_{3}, z_{2} z_{3}^{2}, z_{3}^{2}\right) \\
\operatorname{dim}=6\end{array}$ \\
\hline$E_{7}$ & $\begin{array}{l}e_{1}=-34 x^{5} y^{13}-y x^{17}+34 y^{5} x^{13}+x y^{17} \\
e_{2}=-3 y^{10} x^{2}+6 y^{6} x^{6}-3 y^{2} x^{10} \\
e_{3}=14 y^{4} x^{4}+x^{8}+y^{8}\end{array}$ & $8\left(3 z_{1}^{2}-12 z_{2}^{3}+z_{2} z_{3}^{3}\right)$ & $\begin{array}{l}\operatorname{Vect}\left(1, z_{2}, z_{2}^{2}, z_{3}, z_{2} z_{3}, z_{2}^{2} z_{3}, z_{3}^{2}\right) \\
\operatorname{dim}=7\end{array}$ \\
\hline$E_{8}$ & $\begin{aligned} e_{1}= & x^{30}+522 x^{25} y^{5}-10005 x^{20} y^{10} \\
& -10005 x^{10} y^{20}-522 x^{5} y^{25}+y^{30} \\
e_{2}= & x^{20}-228 x^{15} y^{5}+494 x^{10} y^{10} \\
& +228 x^{5} y^{15}+y^{20} \\
e_{3}= & x^{11} y+11 x^{6} y^{6}-x y^{11}\end{aligned}$ & $10\left(-z_{1}^{2}+z_{2}^{3}+1728 z_{3}^{5}\right)$ & 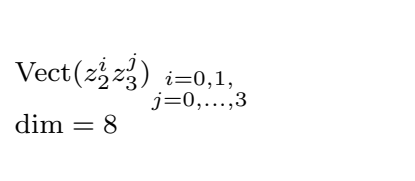 \\
\hline
\end{tabular}

Before carrying on with our study, we make a digression in order to draw a parallel between the Poisson and the Hochschild cohomologies of Klein surfaces, by recalling the result of A. Pichereau.

Theorem 5 (Pichereau). Consider the Poisson bracket defined on $\mathbb{C}\left[z_{1}, z_{1}, z_{3}\right]$ by

$$
\{\cdot, \cdot\}_{f}=\partial_{3} f \partial_{1} \wedge \partial_{2}+\partial_{1} f \partial_{2} \wedge \partial_{3}+\partial_{2} f \partial_{3} \wedge \partial_{1}=i(d f)\left(\partial_{1} \wedge \partial_{2} \wedge \partial_{3}\right)
$$


where $i$ is the contraction of a multiderivation by a differential form. Denote by $H P_{f}^{*}$ (resp. $H P_{*}^{f}$ ) the Poisson cohomology (resp. homology) for this bracket. Under the previous assumptions, the Poisson cohomology $H P_{f}^{*}$ and the Poisson homology $H P_{*}^{f}$ of $\left(\mathbb{C}\left[z_{1}, z_{1}, z_{3}\right] /\langle f\rangle,\{\cdot, \cdot\}_{f}\right)$ are given by

$$
\begin{aligned}
& H P_{f}^{0}=\mathbb{C}, \quad H P_{f}^{1} \simeq H P_{f}^{2}=\{0\}, \\
& H P_{0}^{f} \simeq H P_{2}^{f} \simeq \mathbb{C}\left[z_{1}, z_{2}, z_{3}\right] /\left\langle\partial_{1} f, \partial_{2} f, \partial_{3} f\right\rangle, \\
& \operatorname{dim}\left(H P_{1}^{f}\right)=\operatorname{dim}\left(H P_{0}^{f}\right)-1, \\
& H P_{j}^{f}=H P_{f}^{j}=\{0\} \quad \text { if } j \geq 3 .
\end{aligned}
$$

The algebra $\mathbb{C}[x, y]$ is a Poisson algebra for the standard symplectic bracket $\{\cdot, \cdot\}_{\text {std }}$. As $G$ is a subgroup of the symplectic group $\mathbf{S p}_{2} \mathbb{C}$ ( since $\mathbf{S p}_{2} \mathbb{C}=\mathbf{S L}_{2} \mathbb{C}$ ), the invariant algebra $\mathbb{C}[x, y]^{G}$ is a Poisson subalgebra of $\mathbb{C}[x, y]$. The following proposition allows us to deduce, from Theorem 5 , the Poisson cohomology and homology of $\mathbb{C}[x, y]^{G}$ for the standard symplectic bracket.

Proposition 5. With the choice made in the preceding table for the polynomial $f$, the isomorphism of associative algebras

$$
\pi:\left(\mathbb{C}[x, y]^{G},\{\cdot, \cdot\}_{\mathrm{std}}\right) \rightarrow\left(\mathbb{C}\left[z_{1}, z_{1}, z_{3}\right] /\langle f\rangle,\{\cdot, \cdot\}_{f}\right), \quad e_{j} \mapsto \overline{z_{j}}
$$

is a Poisson isomorphism.

In the sequel, we will calculate the Hochschild cohomology of $\mathbb{C}\left[z_{1}, z_{1}, z_{3}\right] /\langle f\rangle$, and we will immediately deduce the Hochschild cohomology of $\mathbb{C}[x, y]^{G}$, with the help of the isomorphism $\pi$. Note that the fact that $\pi$ preserves the Poisson structures has no incidence on the computation of the Hochschild cohomology. Therefore, so as to simplify the calculations, we may replace the polynomial $f$ by a simpler one, given in the following table

\begin{tabular}{|c||c|c|c|c|c|}
\hline$G$ & $A_{k}$ & $D_{k}$ & $E_{6}$ & $E_{7}$ & $E_{8}$ \\
\hline$f$ & $z_{1}^{2}+z_{2}^{2}+z_{3}^{k}$ & $z_{1}^{2}+z_{2}^{2} z_{3}+z_{3}^{k}$ & $z_{1}^{2}+z_{2}^{3}+z_{3}^{4}$ & $z_{1}^{2}+z_{2}^{3}+z_{2} z_{3}^{3}$ & $z_{1}^{2}+z_{2}^{3}+z_{3}^{5}$ \\
\hline
\end{tabular}

Indeed, the linear maps defined by

$$
\begin{aligned}
& \mathbb{C}[\mathbf{z}] \rightarrow \mathbb{C}[\mathbf{z}] \\
& \left(z_{1}, z_{2}, z_{3}\right) \mapsto\left(\alpha_{1} z_{1}, \alpha_{2} z_{2}, \alpha_{3} z_{3}\right), \\
& \left(z_{1}, z_{2}, z_{3}\right) \mapsto\left(\alpha_{1}\left(z_{1}+z_{2}\right), \alpha_{2}\left(z_{1}+z_{2}\right), \alpha_{3} z_{3}\right)
\end{aligned}
$$

are isomorphisms of associative algebras.

\subsection{Description of the cohomology spaces}

We consider now the case $A:=\mathbb{C}\left[z_{1}, z_{2}, z_{3}\right], /\langle f\rangle$ and we want to calculate the Hochschild cohomology of $A$. We use the notations of Section 2.1, but we change the ordering of the basis: we shall take $\left(\eta_{1} \eta_{2}, \eta_{2} \eta_{3}, \eta_{3} \eta_{1}\right)$ instead of $\left(\eta_{1} \eta_{2}, \eta_{1} \eta_{3}, \eta_{2} \eta_{3}\right)$. The different spaces of the complex are now given by

$$
\begin{aligned}
& \widetilde{T}(0)=A, \\
& \widetilde{T}(1)=A \eta_{1} \oplus A \eta_{2} \oplus A \eta_{3}, \\
& \widetilde{T}(2)=A b_{1} \oplus A \eta_{1} \eta_{2} \oplus A \eta_{2} \eta_{3} \oplus A \eta_{3} \eta_{1}, \\
& \widetilde{T}(3)=A b_{1} \eta_{1} \oplus A b_{1} \eta_{2} \oplus A b_{1} \eta_{3} \oplus A \eta_{1} \eta_{2} \eta_{3},
\end{aligned}
$$


$\widetilde{T}(4)=A b_{1}^{2} \oplus A b_{1} \eta_{1} \eta_{2} \oplus A b_{1} \eta_{2} \eta_{3} \oplus A b_{1} \eta_{3} \eta_{1}$,
$\widetilde{T}(5)=A b_{1}^{2} \eta_{1} \oplus A b_{1}^{2} \eta_{2} \oplus A b_{1}^{2} \eta_{3} \oplus A b_{1} \eta_{1} \eta_{2} \eta_{3}$,

i.e., for an arbitrary $p \in \mathbb{N}^{*}$,

$$
\widetilde{T}(2 p)=A b_{1}^{p} \oplus A b_{1}^{p-1} \eta_{1} \eta_{2} \oplus A b_{1}^{p-1} \eta_{2} \eta_{3} \oplus A b_{1}^{p-1} \eta_{3} \eta_{1},
$$

and

$$
\widetilde{T}(2 p+1)=A b_{1}^{p} \eta_{1} \oplus A b_{1}^{p} \eta_{2} \oplus A b_{1}^{p} \eta_{3} \oplus A b_{1}^{p-1} \eta_{1} \eta_{2} \eta_{3} .
$$

We have

$$
\frac{\partial}{\partial \eta_{1}}\left(\eta_{1} \wedge \eta_{2} \wedge \eta_{3}\right)=1 \wedge \eta_{2} \wedge \eta_{3}=\eta_{2} \wedge \eta_{3} \wedge 1,
$$

thus

$$
d_{\widetilde{T}}^{(3)}\left(\eta_{1} \eta_{2} \eta_{3}\right)=\frac{\partial f}{\partial z_{1}} b_{1} \eta_{2} \eta_{3}+\frac{\partial f}{\partial z_{2}} b_{1} \eta_{3} \eta_{1}+\frac{\partial f}{\partial z_{3}} b_{1} \eta_{1} \eta_{2} .
$$

The matrices of $d_{\widetilde{T}}$ are therefore given by

$$
\begin{aligned}
& \operatorname{Mat}_{\mathcal{B}_{1}, \mathcal{B}_{2}}\left(d_{\widetilde{T}}^{(1)}\right)=\left(\begin{array}{ccc}
\partial_{1} f & \partial_{2} f & \partial_{3} f \\
0 & 0 & 0 \\
0 & 0 & 0 \\
0 & 0 & 0
\end{array}\right), \\
& \forall p \in \mathbb{N}^{*}, \quad \operatorname{Mat}_{\mathcal{B}_{2 p}, \mathcal{B}_{2 p+1}}\left(d_{\widetilde{T}}^{(2 p)}\right)=\left(\begin{array}{cccc}
0 & \partial_{2} f & 0 & -\partial_{3} f \\
0 & -\partial_{1} f & \partial_{3} f & 0 \\
0 & 0 & -\partial_{2} f & \partial_{1} f \\
0 & 0 & 0 & 0
\end{array}\right), \\
& \forall p \in \mathbb{N}^{*}, \quad \operatorname{Mat}_{\mathcal{B}_{2 p+1}, \mathcal{B}_{2 p+2}}\left(d_{\widetilde{T}}^{(2 p+1)}\right)=\left(\begin{array}{cccc}
\partial_{1} f & \partial_{2} f & \partial_{3} f & 0 \\
0 & 0 & 0 & \partial_{3} f \\
0 & 0 & 0 & \partial_{1} f \\
0 & 0 & 0 & \partial_{2} f
\end{array}\right) .
\end{aligned}
$$

We deduce

$$
\begin{aligned}
H^{0} & =A, \\
H^{1} & =\left\{g_{1} \eta_{1}+g_{2} \eta_{2}+g_{3} \eta_{3} /\left(g_{1}, g_{2}, g_{3}\right) \in A^{3} \text { and } g_{1} \partial_{1} f+g_{2} \partial_{2} f+g_{3} \partial_{3} f=0\right\} \\
& \left.\simeq \mathbf{g}=\left(\begin{array}{l}
g_{1} \\
g_{2} \\
g_{3}
\end{array}\right) \in A^{3} / \mathbf{g} \cdot \nabla f=0\right\}, \\
H^{2} & \left.\left.\left.=\frac{\left\{g_{0} b_{1}+g_{3} \eta_{1} \eta_{2}+g_{1} \eta_{2} \eta_{3}+g_{2} \eta_{3} \eta_{1} / \begin{array}{l}
\left(g_{0}, g_{1}, g_{2}, g_{3}\right) \in A^{4} \text { and } \\
g_{2} \partial_{2} f-g_{2} \partial_{3} f=g_{1} \partial_{3} f-g_{3} \partial_{1} f
\end{array}\right\}}{\left\{\left(g_{1} \partial_{1} f+g_{2} \partial_{2} f+g_{3} \partial_{3} f\right) b_{1}, /\left(g_{1}, g_{2}, g_{3}\right) \in A^{3}\right\}} \begin{array}{l}
g_{1} \\
g_{2} \\
g_{3}
\end{array}\right)=0\right\} /\left\{\begin{array}{c}
\mathbf{g} \cdot \nabla f \\
\mathbf{0}_{3,1}
\end{array}\right) / \mathbf{g} \in A^{3}\right\}
\end{aligned}
$$




$$
\simeq \frac{A}{\left\langle\partial_{1} f, \partial_{2} f, \partial_{3} f\right\rangle_{A}} \oplus\left\{\mathbf{g} \in A^{3} / \nabla f \wedge \mathbf{g}=0\right\} .
$$

For $p \geq 2$,

$$
\begin{aligned}
& H^{2 p}=\frac{ \begin{cases}\left.g_{0}, g_{1}, g_{2}, g_{3}\right) \in A^{4} \text { and } \\
\left.g_{0} b_{1}^{p}+g_{3} b_{1}^{p-1} \eta_{1} \eta_{2}+g_{1} b_{1}^{p-1} \eta_{2} \eta_{3}+g_{2} b_{1}^{p-1} \eta_{3} \eta_{1} / \begin{array}{l}
g_{3} \partial_{2} f-g_{2} \partial_{3} f \\
=g_{1} \partial_{3} f-g_{3} \partial_{1} f \\
=g_{2} \partial_{1} f-g_{1} \partial_{2} f=0
\end{array}\right\}\end{cases} }{\left\{\begin{array}{l}
\left(g_{1} \partial_{1} f+g_{2} \partial_{2} f+g_{3} \partial_{3} f\right) b_{1}^{p} \\
+g_{0}\left(\partial_{3} f b_{1}^{p-1} \eta_{1} \eta_{2}+\partial_{1} f b_{1}^{p-1} \eta_{2} \eta_{3}+\partial_{2} f b_{1}^{p-1} \eta_{3} \eta_{1}\right)
\end{array} /\left(g_{0}, g_{1}, g_{2}, g_{3}\right) \in A^{3}\right\}} \\
& \simeq\left\{\mathbf{g}=\left(\begin{array}{c}
g_{0} \\
g_{1} \\
g_{2} \\
g_{3}
\end{array}\right) \in A^{4} / \nabla f \wedge\left(\begin{array}{c}
g_{1} \\
g_{2} \\
g_{3}
\end{array}\right)=0\right\} /\left\{\left(\begin{array}{c}
\mathbf{g} \cdot \nabla f \\
g_{0} \partial_{1} f \\
g_{0} \partial_{2} f \\
g_{0} \partial_{3} f
\end{array}\right) / \mathbf{g} \in A^{3} \text { and } g_{0} \in A\right\} \\
& \simeq \frac{A}{\left\langle\partial_{1} f, \partial_{2} f, \partial_{3} f\right\rangle_{A}} \oplus \frac{\left\{\mathbf{g} \in A^{3} / \nabla f \wedge \mathbf{g}=0\right\}}{\{g \nabla f / g \in A\}} .
\end{aligned}
$$

For $p \in \mathbb{N}^{*}$,

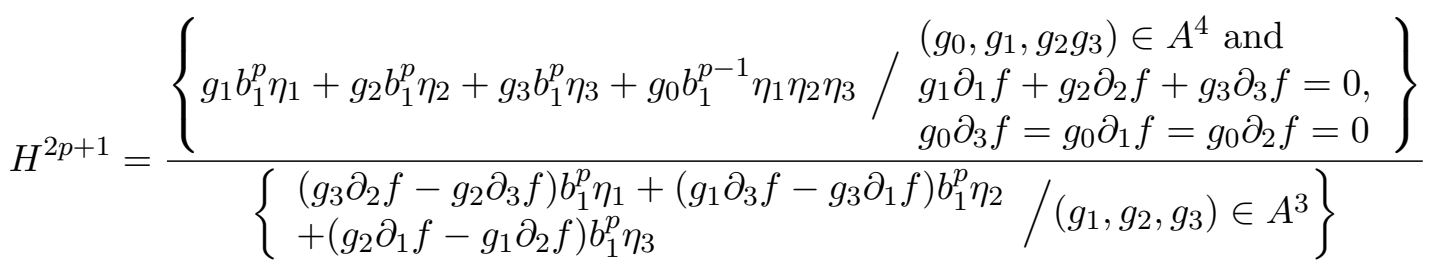

$$
\begin{aligned}
& \simeq\left\{\left(\begin{array}{l}
g_{1} \\
g_{2} \\
g_{3} \\
g_{0}
\end{array}\right) \in A^{4} / \begin{array}{c}
\nabla f \cdot\left(\begin{array}{l}
g_{1} \\
g_{2} \\
g_{3}
\end{array}\right)=0 \\
g_{0} \partial_{3} f=g_{0} \partial_{1} f=g_{0} \partial_{2} f=0
\end{array}\right\} /\left\{\left(\begin{array}{c}
\nabla f \wedge \mathbf{g} \\
0
\end{array}\right) / \mathbf{g} \in A^{3}\right\} \\
& \simeq \frac{\left\{\mathbf{g} \in A^{3} / \nabla f \cdot \mathbf{g}=0\right\}}{\left\{\nabla f \wedge \mathbf{g} / \mathbf{g} \in A^{3}\right\}} \oplus\left\{g \in A / g \partial_{3} f=g \partial_{1} f=g \partial_{2} f=0\right\} .
\end{aligned}
$$

The following section will allow us to make those various spaces more explicit.

\subsection{Explicit calculations in the particular case where $f$ has separate variables}

In this section, we consider the polynomial $f=a_{1} z_{1}^{i}+a_{2} z_{2}^{j}+a_{3} z_{3}^{k}$, with $2 \leq i \leq j \leq k$ and $a_{j} \in \mathbb{C}^{*}$. Its partial derivatives are $\partial_{1} f=i a_{1} z_{1}^{i-1}, \partial_{2} f=j a_{2} z_{2}^{j-1}$ and $\partial_{3} f=k a_{3} z_{3}^{k-1}$.

We already have

$$
H^{0}=\mathbb{C}\left[z_{1}, z_{2}, z_{3}\right] /\left\langle a_{1} z_{1}^{i}+a_{2} z_{2}^{j}+a_{3} z_{3}^{k}\right\rangle .
$$

Moreover, as $f$ is weighted homogeneous, Euler's formula gives

$$
\frac{1}{i} z_{1} \partial_{1} f+\frac{1}{j} z_{2} \partial_{2} f+\frac{1}{k} z_{3} \partial_{3} f=f .
$$

So we have the inclusion $\langle f\rangle \subset\left\langle\partial_{1} f, \partial_{2} f, \partial_{3} f\right\rangle$, thus

$$
\begin{aligned}
\frac{A}{\left\langle\partial_{1} f, \partial_{2} f, \partial_{3} f\right\rangle_{A}} & \simeq \frac{\mathbb{C}\left[z_{1}, z_{2}, z_{3}\right]}{\left\langle\partial_{1} f, \partial_{2} f, \partial_{3} f\right\rangle} \\
& \simeq \operatorname{Vect}\left(z_{1}^{p} z_{2}^{q} z_{3}^{r} / p \in \llbracket 0, i-2 \rrbracket, q \in \llbracket 0, j-2 \rrbracket, r \in \llbracket 0, k-2 \rrbracket\right) .
\end{aligned}
$$


Finally, as $\partial_{1} f$ and $f$ are relatively prime, if $g \in A$ verifies $g \partial_{1} f=0 \bmod \langle f\rangle$, then $g \in\langle f\rangle$, i.e. $g$ is zero in $A$.

Now we determine the set

$$
\left\{\mathbf{g}=\left(\begin{array}{c}
g_{1} \\
g_{2} \\
g_{3}
\end{array}\right) \in A^{3} / \mathbf{g} \cdot \nabla f=0\right\}
$$

First we have

$$
\left\langle f, \partial_{1} f, \partial_{2} f\right\rangle=\left\langle a_{1} z_{1}^{i}+a_{2} z_{2}^{j}+a_{3} z_{3}^{k}, z_{1}^{i-1}, z_{2}^{j-1}\right\rangle=\left\langle z_{1}^{i-1}, z_{2}^{j-1}, z_{3}^{k}\right\rangle .
$$

Thus the only monomials which are not in this ideal are the elements $z_{1}^{p} z_{2}^{q} z_{3}^{r}$ with $p \in \llbracket 0, i-2 \rrbracket$, $q \in \llbracket 0, j-2 \rrbracket$, and $r \in \llbracket 0, k-1 \rrbracket$.

So every polynomial $P \in \mathbb{C}[\mathbf{z}]$ may be written in the form

$$
P=\alpha f+\beta \partial_{1} f+\gamma \partial_{2} f+\sum_{\substack{p=0, \ldots, i-2 \\ q=0, \ldots, j-2 \\ r=0, \ldots, k-1}} a_{p q r} z_{1}^{p} z_{2}^{q} z_{3}^{r} .
$$

The polynomials $P \in \mathbb{C}[\mathbf{z}]$ such that $P \partial_{3} f \in\left\langle f, \partial_{1} f, \partial_{2} f\right\rangle$ are therefore the following ones

$$
P=\alpha f+\beta \partial_{1} f+\gamma \partial_{2} f+\sum_{\substack{p=0, \ldots, i-2 \\ q=0, \ldots, j-2 \\ r=1, \ldots, k-1}} a_{p q r} z_{1}^{p} z_{2}^{q} z_{3}^{r} .
$$

So we have calculated $\operatorname{Ann}_{\left\langle f, \partial_{1} f, \partial_{2} f\right\rangle}\left(\partial_{3} f\right)$. The equation

$$
\mathbf{g} \cdot \nabla f=0 \bmod \langle f\rangle
$$

leads to $g_{3} \in \operatorname{Ann}_{\left\langle f, \partial_{1} f, \partial_{2} f\right\rangle}\left(\partial_{3} f\right)$, i.e.

$$
g_{3}=\alpha f+\beta \partial_{1} f+\gamma \partial_{2} f+\sum_{\substack{p=0, \ldots, i-2 \\ q=0, \ldots, j-2 \\ r=1 \ldots k-1}} a_{p q r} z_{1}^{p} z_{2}^{q} z_{3}^{r}
$$

with $(\alpha, \beta, \gamma) \in \mathbb{C}[\mathbf{z}]^{3}$. Hence

$$
g_{2} \partial_{2} f+\gamma \partial_{2} f \partial_{3} f+\sum_{\substack{p=0, \ldots, i-2 \\ q=0, \ldots, j-2 \\ r=1, \ldots, k-1}} a_{p q r} z_{1}^{p} z_{2}^{q} z_{3}^{r} \partial_{3} f \in\left\langle f, \partial_{1} f\right\rangle
$$

Thus, according to Euler's formula,

$$
\partial_{2} f\left(g_{2}+\gamma \partial_{3} f-\frac{k}{j} \sum_{\substack{p=0, \ldots, i-2 \\ q=0, \ldots j-2 \\ r=1, \ldots, k-1}} a_{p q r} z_{1}^{p} z_{2}^{q+1} z_{3}^{r-1}\right) \in\left\langle f, \partial_{1} f\right\rangle .
$$

Since $\operatorname{Ann}_{\left\langle f, \partial_{1} f\right\rangle}\left(\partial_{2} f\right)=\left\langle f, \partial_{1} f\right\rangle$, this equation is equivalent to

$$
g_{2}=-\gamma \partial_{3} f+\frac{k}{j} \sum_{\begin{array}{c}
p=0, \ldots, i-2 \\
q=0, \ldots,-2 \\
r=1, \ldots, k-1
\end{array}} a_{p q r} z_{1}^{p} z_{2}^{q+1} z_{3}^{r-1}+\delta f+\varepsilon \partial_{1} f
$$


with $\delta, \varepsilon \in \mathbb{C}[\mathbf{z}]$. It follows that

$$
\begin{aligned}
& g_{1} \partial_{1} f+\beta \partial_{1} f \partial_{3} f+\varepsilon \partial_{1} f \partial_{2} f \\
& \quad+\sum_{\substack{p=0, \ldots, i-2 \\
q=0, \ldots, j-2 \\
r=1, \ldots, k-1}} a_{p q r} z_{1}^{p} z_{2}^{q} z_{3}^{r} \partial_{3} f+\frac{k}{j} \sum_{\substack{p=0, \ldots, i-2 \\
q=0, \ldots, j-2 \\
r=1, \ldots, k-1}} a_{p q r} z_{1}^{p} z_{2}^{q+1} z_{3}^{r-1} \partial_{2} f \in\langle f\rangle .
\end{aligned}
$$

And, according to Euler's formula,

$$
\partial_{1} f\left(g_{1}+\beta \partial_{3} f+\varepsilon \partial_{2} f-\frac{k}{i} \sum_{\begin{array}{c}
p=0, \ldots, i-2 \\
q=0, \ldots, j-2 \\
r=1, \ldots, k-1
\end{array}} a_{p q r} z_{1}^{p+1} z_{2}^{q} z_{3}^{r-1}\right) \in\langle f\rangle,
$$

i.e.

$$
g_{1}=-\beta \partial_{3} f-\varepsilon \partial_{2} f+\frac{k}{i} \sum_{\substack{p=0, \ldots, i-2 \\ q=0, \ldots, j-2 \\ r=1, \ldots, k-1}} a_{p q r} z_{1}^{p+1} z_{2}^{q} z_{3}^{r-1}+\eta f
$$

with $\eta \in \mathbb{C}[\mathbf{z}]$. Finally

$$
\begin{aligned}
\{\mathbf{g} & \left.\in A^{3} / \mathbf{g} \cdot \nabla f=0\right\} \\
& =\left\{\nabla f \wedge\left(\begin{array}{c}
-\gamma \\
\beta \\
-\varepsilon
\end{array}\right)+\sum_{\substack{p=0, \ldots, i-2 \\
q=0, \ldots, j-2 \\
r=1, \ldots, k-1}} a_{p q r} z_{1}^{p} z_{2}^{q} z_{3}^{r-1}\left(\begin{array}{c}
\frac{k}{i} z_{1} \\
\frac{k}{j} z_{2} \\
z_{3}
\end{array}\right) /(\beta, \gamma, \varepsilon) \in A^{3} \text { and } a_{p q r} \in \mathbb{C}\right\} .
\end{aligned}
$$

We deduce immediately the cohomology spaces of odd degrees

$$
\begin{aligned}
& \forall p \geq 1, \quad H^{2 p+1} \simeq \mathbb{C}^{(i-1)(j-1)(k-1)}, \\
& H^{1} \simeq \nabla f \wedge(\mathbb{C}[\mathbf{z}] /\langle f\rangle)^{3} \oplus \mathbb{C}^{(i-1)(j-1)(k-1)} .
\end{aligned}
$$

It remains to determine the set

$$
\left\{\mathbf{g}=\left(\begin{array}{l}
g_{1} \\
g_{2} \\
g_{3}
\end{array}\right) \in A^{3} / \nabla f \wedge \mathbf{g}=0\right\} .
$$

Let $\mathbf{g} \in A^{3}$ be such that $\nabla f \wedge \mathbf{g}=0$. This means that, modulo $\langle f\rangle$, $\mathbf{g}$ verifies the system

$$
\partial_{2} f g_{3}-\partial_{3} f g_{2}=0, \quad \partial_{3} f g_{1}-\partial_{1} f g_{3}=0, \quad \partial_{1} f g_{2}-\partial_{2} f g_{1}=0 .
$$

The first equation gives, modulo $\left\langle f, \partial_{2} f\right\rangle, \partial_{3} f g_{2}=0$. Now $\operatorname{Ann}_{\left\langle f, \partial_{2} f\right\rangle}\left(\partial_{3} f\right)=\left\langle f, \partial_{2} f\right\rangle$, therefore $g_{2}=\alpha f+\beta \partial_{2} f$. Hence

$$
\partial_{2} f\left(g_{3}-\beta \partial_{3} f\right)=0 \bmod \langle f\rangle \text {, }
$$

i.e. $g_{3}=\gamma f+\beta \partial_{3} f$. Finally, we obtain

$$
\partial_{3} f\left(g_{1}-\beta \partial_{1} f\right)=0 \bmod \langle f\rangle,
$$

i.e. $g_{1}=\delta f+\beta \partial_{1} f$. So, $\left\{\mathbf{g} \in A^{3} / \nabla f \wedge \mathbf{g}=0\right\}=\{\beta \nabla f / \beta \in A\}$. 
We deduce the cohomology spaces of even degrees (for the direct sum, we use the same argument as in Section 3.2)

$$
\begin{aligned}
\forall p \geq 2, \quad H^{2 p} & \simeq A /\left\langle\partial_{1} f, \partial_{2} f, \partial_{3} f\right\rangle \simeq \mathbb{C}[\mathbf{z}] /\left\langle z_{1}^{i-1}, z_{2}^{j-1}, z_{3}^{k-1}\right\rangle \\
& \simeq \operatorname{Vect}\left(z_{1}^{p} z_{2}^{q} z_{3}^{r} / p \in \llbracket 0, i-2 \rrbracket, q \in \llbracket 0, j-2 \rrbracket, r \in \llbracket 0, k-2 \rrbracket\right) \\
& \simeq \mathbb{C}^{(i-1)(j-1)(k-1)}, \\
H^{2} & \simeq\{\beta \nabla f / \beta \in A\} \oplus \mathbb{C}^{(i-1)(j-1)(k-1)} \\
& \simeq \mathbb{C}[\mathbf{z}] /\left\langle a_{1} z_{1}^{i}+a_{2} z_{2}^{j}+a_{3} z_{3}^{k}\right\rangle \oplus \mathbb{C}^{(i-1)(j-1)(k-1)} .
\end{aligned}
$$

Remark 4. We also have

$$
\nabla f \wedge(\mathbb{C}[\mathbf{z}] /\langle f\rangle)^{3} \simeq(\mathbb{C}[\mathbf{z}] /\langle f\rangle)^{3} /\{\mathbf{g} / \nabla f \wedge \mathbf{g}=0\}=(\mathbb{C}[\mathbf{z}] /\langle f\rangle)^{3} /(\mathbb{C}[\mathbf{z}] /\langle f\rangle) \nabla f .
$$

Moreover the map

$$
(\mathbb{C}[\mathbf{z}] /\langle f\rangle)^{2} \rightarrow \nabla f \wedge(\mathbb{C}[\mathbf{z}] /\langle f\rangle)^{3}, \quad\left(\begin{array}{c}
g_{1} \\
g_{2}
\end{array}\right) \mapsto \nabla f \wedge\left(\begin{array}{c}
g_{1} \\
g_{2} \\
0
\end{array}\right)
$$

is injective, thus $\nabla f \wedge(\mathbb{C}[\mathbf{z}] /\langle f\rangle)^{3}$ is infinite-dimensional.

Remark 5. In particular, we obtain the cohomology for the cases where $f=z_{1}^{2}+z_{2}^{2}+z_{3}^{k}$, $f=z_{1}^{2}+z_{2}^{3}+z_{3}^{4}$ and $f=z_{1}^{2}+z_{2}^{3}+z_{3}^{5}$. These cases correspond respectively to the types $A_{k}, E_{6}$ and $E_{8}$ of the Klein surfaces.

The following table sums up the results of those three special cases:

\begin{tabular}{|l||l|l|l|l|}
\hline & $H^{0}$ & $H^{1}$ & $H^{2}$ & $H^{p}, p \geq 3$ \\
\hline \hline$A_{k}$ & $\mathbb{C}[\mathbf{z}] /\left\langle z_{1}^{2}+z_{2}^{2}+z_{3}^{k}\right\rangle$ & $\nabla f \wedge(\mathbb{C}[\mathbf{z}] /\langle f\rangle)^{3} \oplus \mathbb{C}^{k-1}$ & $\mathbb{C}[\mathbf{z}] /\left\langle z_{1}^{2}+z_{2}^{2}+z_{3}^{k}\right\rangle \oplus \mathbb{C}^{k-1}$ & $\mathbb{C}^{k-1}$ \\
\hline$E_{6}$ & $\mathbb{C}[\mathbf{z}] /\left\langle z_{1}^{2}+z_{2}^{3}+z_{3}^{4}\right\rangle$ & $\nabla f \wedge(\mathbb{C}[\mathbf{z}] /\langle f\rangle)^{3} \oplus \mathbb{C}^{6}$ & $\mathbb{C}[\mathbf{z}] /\left\langle z_{1}^{2}+z_{2}^{3}+z_{3}^{4}\right\rangle \oplus \mathbb{C}^{6}$ & $\mathbb{C}^{6}$ \\
\hline$E_{8}$ & $\mathbb{C}[\mathbf{z}] /\left\langle z_{1}^{2}+z_{2}^{3}+z_{3}^{5}\right\rangle$ & $\nabla f \wedge(\mathbb{C}[\mathbf{z}] /\langle f\rangle)^{3} \oplus \mathbb{C}^{8}$ & $\mathbb{C}[\mathbf{z}] /\left\langle z_{1}^{2}+z_{2}^{3}+z_{3}^{5}\right\rangle \oplus \mathbb{C}^{8}$ & $\mathbb{C}^{8}$ \\
\hline
\end{tabular}

The cases where $f=z_{1}^{2}+z_{2}^{2} z_{3}+z_{3}^{k}$ and $f=z_{1}^{2}+z_{2}^{3}+z_{2} z_{3}^{3}$, i.e. respectively $D_{k}$ and $E_{7}$ are studied in the following section.

\subsection{Explicit calculations for $D_{k}$ and $E_{7}$}

\subsubsection{Case of $f=z_{1}^{2}+z_{2}^{2} z_{3}+z_{3}^{k}$, i.e. $D_{k}$}

In this section, we consider the polynomial $f=z_{1}^{2}+z_{2}^{2} z_{3}+z_{3}^{k}$, with $k \geq 3$. Its partial derivatives are $\partial_{1} f=2 z_{1}, \partial_{2} f=2 z_{2} z_{3}$ and $\partial_{3} f=z_{2}^{2}+k z_{3}^{k-1}$.

We already have

$$
H^{0}=\mathbb{C}[\mathbf{z}] /\left\langle z_{1}^{2}+z_{2}^{2} z_{3}+z_{3}^{k}\right\rangle .
$$

Besides, since $f$ is weighted homogeneous, Euler's formula gives

$$
\frac{k}{2} z_{1} \partial_{1} f+\frac{k-1}{2} z_{2} \partial_{2} f+z_{3} \partial_{3} f=k f .
$$

Thus, we have the inclusion $\langle f\rangle \subset\left\langle\partial_{1} f, \partial_{2} f, \partial_{3} f\right\rangle$. Moreover, a Groebner basis of $\left\langle\partial_{1} f, \partial_{2} f, \partial_{3} f\right\rangle$ is $\left[z_{3}^{k}, z_{2} z_{3}, z_{2}^{2}+k z_{3}^{k-1}, z_{1}\right]$, therefore

$$
\frac{A}{\left\langle\partial_{1} f, \partial_{2} f, \partial_{3} f\right\rangle_{A}} \simeq \frac{\mathbb{C}\left[z_{1}, z_{2}, z_{3}\right]}{\left\langle\partial_{1} f, \partial_{2} f, \partial_{3} f\right\rangle} \simeq \operatorname{Vect}\left(z_{2}, 1, z_{3}, \ldots, z_{3}^{k-1}\right) .
$$


Finally, as $\partial_{1} f$ and $f$ are relatively prime, if $g \in A$ verifies $g \partial_{1} f=0 \bmod \langle f\rangle$, then $g \in\langle f\rangle$, i.e. $g$ is zero in $A$, thus $\left\{g \in A / g \partial_{3} f=g \partial_{1} f=g \partial_{2} f=0\right\}=0$.

Now we determine the set

$$
\left\{\mathbf{g}=\left(\begin{array}{c}
g_{1} \\
g_{2} \\
g_{3}
\end{array}\right) \in A^{3} / \mathbf{g} \cdot \nabla f=0\right\} .
$$

A Groebner basis of $\left\langle f, \partial_{1} f, \partial_{3} f\right\rangle$ is $\left[z_{1}, z_{3}^{k}, z_{2}^{2}+k z_{3}^{k-1}\right]$, thus a basis of $\mathbb{C}[\mathbf{z}] /\left\langle f, \partial_{1} f, \partial_{3} f\right\rangle$ is $\left\{z_{2}^{i} z_{3}^{j} / i \in\{0,1\}, j \in \llbracket 0, k-1 \rrbracket\right\}$. We have already solved the equation $p \partial_{2} f=0$ in this space; the solutions of this equation in $\mathbb{C}[\mathbf{z}] /\left\langle f, \partial_{1} f, \partial_{3} f\right\rangle$ are of the form

$$
p=a_{0, k-1} z_{3}^{k-1}+\sum_{j=0}^{k-1} a_{1, j} z_{2} z_{3}^{j}
$$

where $a_{0, k-1}, a_{1, j} \in \mathbb{C}$.

Let $\mathbf{g}=\left(\begin{array}{c}g_{1} \\ g_{2} \\ g_{3}\end{array}\right) \in A^{3}$ satisfy the equation

$$
\mathbf{g} \cdot \nabla f=0 \bmod \langle f\rangle .
$$

Then we have

$$
g_{2} \partial_{2} f=0 \bmod \left\langle f, \partial_{1} f, \partial_{3} f\right\rangle,
$$

hence

$$
g_{2}=\alpha f+\beta \partial_{1} f+\gamma \partial_{3} f+a z_{3}^{k-1}+\sum_{j=0}^{k-1} b_{j} z_{2} z_{3}^{j},
$$

with $(\alpha, \beta, \gamma) \in \mathbb{C}[\mathbf{z}]^{3}$. And

$$
g_{3} \partial_{3} f+\gamma \partial_{3} f \partial_{2} f+a z_{3}^{k-1} \partial_{2} f+\sum_{j=0}^{k-1} b_{j} z_{2} z_{3}^{j} \partial_{2} f \in\left\langle f, \partial_{1} f\right\rangle .
$$

Now according to Euler's formula (3) and the equality

$$
z_{3}^{k} z_{2}=\frac{1}{1-k}\left(z_{2} f-z_{2} z_{3} \partial_{3} f-\frac{1}{2} z_{2} z_{1} \partial_{1} f\right)=-\frac{1}{1-k} z_{2} z_{3} \partial_{3} f \quad \bmod \left\langle f, \partial_{1} f\right\rangle
$$

Equation (4) becomes

$$
\partial_{3} f\left(g_{3}+\gamma \partial_{2} f-\frac{2 a}{1-k} z_{2} z_{3}-\sum_{j=0}^{k-1} b_{j} \frac{2}{k-1} z_{3}^{j+1}\right) \in\left\langle f, \partial_{1} f\right\rangle .
$$

As $\operatorname{Ann}_{\left\langle f, \partial_{1} f\right\rangle}\left(\partial_{3} f\right)=\left\langle f, \partial_{1} f\right\rangle$, this equation is equivalent to

$$
g_{3}=-\gamma \partial_{2} f+\frac{2 a}{1-k} z_{2} z_{3}+\sum_{j=0}^{k-1} b_{j} \frac{2}{k-1} z_{3}^{j+1}+\delta f+\varepsilon \partial_{1} f,
$$


with $\delta, \varepsilon \in \mathbb{C}[\mathbf{z}]$. We find

$$
g_{1}=-\beta \partial_{2} f-\varepsilon \partial_{3} f+\sum_{j=0}^{k-1} b_{j} \frac{k}{k-1} z_{1} z_{3}^{j}+\frac{a}{1-k} z_{2} z_{1}+\eta f,
$$

with $\eta \in \mathbb{C}[\mathbf{z}]$. Finally, we have

$$
\begin{aligned}
& \left\{\mathbf{g} \in A^{3} / \mathbf{g} \cdot \nabla f=0\right\} \\
& \quad=\left\{\nabla f \wedge\left(\begin{array}{c}
\gamma \\
\varepsilon \\
-\beta
\end{array}\right)+\sum_{j=0}^{k-1} b_{j}\left(\begin{array}{c}
\frac{k}{k-1} z_{1} z_{3}^{j} \\
z_{2} z_{3}^{j} \\
-\frac{2}{1-k} z_{3}^{j+1}
\end{array}\right)+a\left(\begin{array}{c}
\frac{1}{1-k} z_{2} z_{1} \\
z_{3}^{k-1} \\
\frac{2}{1-k} z_{2} z_{3}
\end{array}\right) / \begin{array}{l}
(\beta, \gamma, \varepsilon) \in A^{3} \\
\text { and } a, b_{j} \in \mathbb{C}
\end{array}\right\},
\end{aligned}
$$

as well as cohomology spaces of odd degrees (for the direct sum, we use the same argument as in Section 3.2)

$$
\begin{aligned}
& \forall p \geq 1, \quad H^{2 p+1} \simeq \mathbb{C}^{k+1}, \\
& H^{1} \simeq \nabla f \wedge(\mathbb{C}[\mathbf{z}] /\langle f\rangle)^{3} \oplus \mathbb{C}^{k+1} .
\end{aligned}
$$

To show $\left\{\mathbf{g} \in A^{3} / \nabla f \wedge \mathbf{g}=0\right\}=\left\{f \mathbf{g}+\beta \nabla f / \mathbf{g} \in A^{3}, \beta \in A\right\}$, we proceed as in the case of separate variables. We deduce the cohomology spaces of even degrees

$$
\begin{aligned}
\forall p \geq 2, \quad H^{2 p} \simeq A /\left\langle\partial_{1} f, \partial_{2} f, \partial_{3} f\right\rangle \simeq \operatorname{Vect}\left(z_{2}, 1, z_{3}, \ldots, z_{3}^{k-1}\right) \simeq \mathbb{C}^{k+1} \\
H^{2} \simeq\{\beta \nabla f / \beta \in A\} \oplus \mathbb{C}^{k+1} \simeq \mathbb{C}[\mathbf{z}] /\left\langle z_{1}^{2}+z_{2}^{2} z_{3}+z_{3}^{k}\right\rangle \oplus \mathbb{C}^{k+1}
\end{aligned}
$$

\subsubsection{Case of $f=z_{1}^{2}+z_{2}^{3}+z_{2} z_{3}^{3}$, i.e. $E_{7}$}

Here we have $\partial_{1} f=2 z_{1}, \partial_{2} f=3 z_{2}^{2}+z_{3}^{3}$ and $\partial_{3} f=3 z_{2} z_{3}^{2}$. The proof is similar to that of the previous cases. A Groebner basis of $\left\langle\partial_{1} f, \partial_{2} f, \partial_{3} f\right\rangle$ is $\left[z_{3}^{5}, z_{2} z_{3}^{2}, 3 z_{2}^{2}+z_{3}^{3}, z_{1}\right]$. Similarly, a Groebner basis of $\left\langle f, \partial_{1} f, \partial_{2} f\right\rangle$ is $\left[z_{3}^{6}, z_{2} z_{3}^{3}, 3 z_{2}^{2}+z_{3}^{3}, z_{1}\right]$. We obtain the following results

$$
\begin{array}{ll}
\forall p \geq 1, & H^{2 p+1} \simeq \mathbb{C}^{7}, \\
& H^{1} \simeq \nabla f \wedge(\mathbb{C}[\mathbf{z}] /\langle f\rangle)^{3} \oplus \mathbb{C}^{7}, \\
\forall p \geq 2, & H^{0}=\mathbb{C}[\mathbf{z}] /\left\langle z_{1}^{2}+z_{2}^{3}+z_{2} z_{3}^{3}\right\rangle, \\
& H^{2 p} \simeq A /\left\langle\partial_{1} f, \partial_{2} f, \partial_{3} f\right\rangle \simeq \operatorname{Vect}\left(z_{2}, z_{2}^{2}, 1, z_{3}, z_{3}^{2}, z_{3}^{3}, z_{3}^{4}\right) \simeq \mathbb{C}^{7}, \\
& H^{2} \simeq\{\beta \nabla f / \beta \in A\} \oplus \mathbb{C}^{7} \simeq \mathbb{C}[\mathbf{z}] /\left\langle z_{1}^{2}+z_{2}^{3}+z_{2} z_{3}^{3}\right\rangle \oplus \mathbb{C}^{7} .
\end{array}
$$

Remark 6. In all the previously studied cases, there exists a triple $(i, j, k)$ such that $\{i, j, k\}=$ $\{1,2,3\}$, and such that the map

$$
\begin{aligned}
& \mathbb{C}[\mathbf{z}] /\left\langle\partial_{1} f, \partial_{2} f, \partial_{3} f\right\rangle \rightarrow\left\{g \in \mathbb{C}[\mathbf{z}] /\left\langle f, \partial_{j} f, \partial_{k} f\right\rangle / g \partial_{i} f=0\right\}, \\
& P \bmod \left\langle\partial_{1} f, \partial_{2} f, \partial_{3} f\right\rangle \mapsto z_{i} P \bmod \left\langle f, \partial_{j} f, \partial_{k} f\right\rangle
\end{aligned}
$$

is an isomorphism of vector spaces.

\subsection{Homology}

The study is the same as the one of the Hochschild cohomology, and we proceed as in Section 3.5. Here, we have

$$
\widetilde{\Omega}(0)=A, \quad \widetilde{\Omega}(-1)=A \xi_{1} \oplus A \xi_{2} \oplus A \xi_{3},
$$




$$
\begin{array}{ll}
\forall p \in \mathbb{N}^{*}, \quad \widetilde{\Omega}(-2 p)=A a_{1}^{p} \oplus A a_{1}^{p-1} \xi_{1} \xi_{2} \oplus A a_{1}^{p-1} \xi_{2} \xi_{3} \oplus A a_{1}^{p-1} \xi_{3} \xi_{1}, \\
\forall p \in \mathbb{N}^{*}, \quad \widetilde{\Omega}(-2 p-1)=A a_{1}^{p} \xi_{1} \oplus A a_{1}^{p} \xi_{2} \oplus A a_{1}^{p} \xi_{3} \oplus A a_{1}^{p-1} \xi_{1} \xi_{2} \xi_{3} .
\end{array}
$$

This defines the bases $\mathcal{V}_{p}$. The differential is $d_{\widetilde{\Omega}}=\left(\xi_{1} \partial_{1} f+\xi_{2} \partial_{2} f+\xi_{3} \partial_{3} f\right) \frac{\partial}{\partial a_{1}}$.

By setting $D f:=\left(\partial_{3} f \partial_{1} f \partial_{2} f\right)$, we deduce the matrices

$$
\begin{gathered}
\operatorname{Mat}_{\mathcal{V}_{-2}, \mathcal{V}_{-1}}\left(d_{\widetilde{\Omega}}^{(-2)}\right)=\left(\begin{array}{ll}
\nabla f & \mathbf{0}_{3,3}
\end{array}\right), \\
\forall p \geq 2, \quad \operatorname{Mat}_{\mathcal{V}_{-2 p}, \mathcal{V}_{-2 p+1}}\left(d_{\widetilde{\Omega}}^{(-2 p)}\right)=\left(\begin{array}{cc}
\nabla f & \mathbf{0}_{3,3} \\
0 & (p-1) D f
\end{array}\right), \\
\forall p \geq 1, \quad \operatorname{Mat}_{\mathcal{V}_{-2 p-1}, \mathcal{V}_{-2 p}}\left(d_{\widetilde{\Omega}}^{(-2 p-1)}\right)=\left(\begin{array}{cccc}
0 & 0 & 0 & 0 \\
-p \partial_{2} f & p \partial_{1} f & 0 & 0 \\
0 & -p \partial_{3} f & p \partial_{2} f & 0 \\
p \partial_{3} f & 0 & -p \partial_{1} f & 0
\end{array}\right) .
\end{gathered}
$$

The cohomology spaces read as

$$
\begin{aligned}
& L^{0}=A, \quad L^{-1}=\frac{A^{3}}{\{g \nabla f / g \in A\}}, \\
& L^{-2}=\left\{g \in A / g \partial_{1} f=g \partial_{2} f=g \partial_{3} f=0\right\} \oplus \frac{A^{3}}{\left\{\nabla f \wedge \mathbf{g} / \mathbf{g} \in A^{3}\right\}} .
\end{aligned}
$$

For $p \geq 2$,

$$
L^{-2 p} \simeq\left\{g \in A / g \partial_{1} f=g \partial_{2} f=g \partial_{3} f=0\right\} \oplus \frac{\left\{\mathbf{g} \in A^{3} / \mathbf{g} \cdot \nabla f=0\right\}}{\left\{\nabla f \wedge \mathbf{g} / \mathbf{g} \in A^{3}\right\}} .
$$

For $p \in \mathbb{N}^{*}$,

$$
L^{-2 p-1} \simeq \frac{\left\{\mathbf{g} \in A^{3} / \nabla f \wedge \mathbf{g}=0\right\}}{\{g \nabla f / g \in A\}} \oplus \frac{A}{\langle\nabla f\rangle_{A}} .
$$

From now on, we assume that either $f$ has separate variables, or $f$ is of type $D_{k}$ or $E_{7}$. Then we have $\left\{g \in A / g \partial_{1} f=g \partial_{2} f=g \partial_{3} f=0\right\}=\{0\}$, and according to Euler's formula,

$$
\frac{A}{\langle\nabla f\rangle_{A}} \simeq \frac{\mathbb{C}[\mathbf{z}]}{\langle\nabla f\rangle}
$$

Most of the spaces have already been computed in Sections 4.3 and 4.4. In particular, we have $A^{3} / A \nabla f \simeq \nabla f \wedge A^{3}$. Moreover, $\left\{\nabla f \wedge \mathbf{g} / \mathbf{g} \in A^{3}\right\} \subset\left\{\mathbf{g} \in A^{3} / \mathbf{g} \cdot \nabla f=0\right\}$, thus

$$
\operatorname{dim}\left(A^{3} /\left\{\nabla f \wedge \mathbf{g} / \mathbf{g} \in A^{3}\right\}\right) \geq \operatorname{dim}\left(A^{3} /\left\{\mathbf{g} \in A^{3} / \mathbf{g} \cdot \nabla f=0\right\}\right) .
$$

And $A^{3} /\left\{\mathbf{g} \in A^{3} / \mathbf{g} \cdot \nabla f=0\right\} \simeq\left\{\mathbf{g} \cdot \nabla f / \mathbf{g} \in A^{3}\right\}$. Since the map

$$
g \in A \mapsto\left(\begin{array}{l}
g \\
0 \\
0
\end{array}\right) \cdot \nabla f \in\left\{\mathbf{g} \cdot \nabla f / \mathbf{g} \in A^{3}\right\}
$$

is injective, $A^{3} /\left\{\nabla f \wedge \mathbf{g} / \mathbf{g} \in A^{3}\right\}$ is infinite-dimensional.

In the following table we collect the results for the Hochschild homology in the various cases

\begin{tabular}{|l||l|l|l|l|}
\hline Type & $H H_{0}=A$ & $H H_{1}$ & $H H_{2}$ & $H H_{p}, p \geq 3$ \\
\hline \hline$A_{k}$ & $\mathbb{C}[\mathbf{z}] /\left\langle z_{1}^{2}+z_{2}^{2}+z_{3}^{k}\right\rangle$ & $\nabla f \wedge A^{3}$ & $A^{3} /\left(\nabla f \wedge A^{3}\right)$ & $\mathbb{C}^{k-1}$ \\
\hline$D_{k}$ & $\mathbb{C}[\mathbf{z}] /\left\langle z_{1}^{2}+z_{2}^{2} z_{3}+z_{3}^{k}\right\rangle$ & $\nabla f \wedge A^{3}$ & $A^{3} /\left(\nabla f \wedge A^{3}\right)$ & $\mathbb{C}^{k+1}$ \\
\hline$E_{6}$ & $\mathbb{C}[\mathbf{z}] /\left\langle z_{1}^{2}+z_{2}^{3}+z_{3}^{4}\right\rangle$ & $\nabla f \wedge A^{3}$ & $A^{3} /\left(\nabla f \wedge A^{3}\right)$ & $\mathbb{C}^{6}$ \\
\hline$E_{7}$ & $\mathbb{C}[\mathbf{z}] /\left\langle z_{1}^{2}+z_{2}^{3}+z_{2} z_{3}^{3}\right\rangle$ & $\nabla f \wedge A^{3}$ & $A^{3} /\left(\nabla f \wedge A^{3}\right)$ & $\mathbb{C}^{7}$ \\
\hline$E_{8}$ & $\mathbb{C}[\mathbf{z}] /\left\langle z_{1}^{2}+z_{2}^{3}+z_{3}^{5}\right\rangle$ & $\nabla f \wedge A^{3}$ & $A^{3} /\left(\nabla f \wedge A^{3}\right)$ & $\mathbb{C}^{8}$ \\
\hline
\end{tabular}




\section{Acknowledgements}

I would like to thank my thesis advisors Gadi Perets and Claude Roger for their efficient and likeable help, for their great availability, and for the time that they devoted to me all along this study. I also thank Daniel Sternheimer who paid attention to my work and the referees for their relevant remarks and their judicious advice. And I am grateful to Serge Parmentier for the rereading of my English text.

\section{References}

[1] Alev J., Farinati M.A., Lambre T., Solotar A. L., Homologie des invariants d'une algèbre de Weyl sous l'action d'un groupe fini, J. Algebra 232 (2000), 564-577.

[2] Alev J., Lambre T., Comparaison de l'homologie de Hochschild et de l'homologie de Poisson pour une déformation des surfaces de Klein, in Algebra and Operator Theory (Tashkent, 1997), Kluwer Acad. Publ., Dordrecht, 1998, 25-38.

[3] Arnold V., Varchenko A., Goussein-Zadé S., Singularités des applications différentiables, première partie, Mir, Moscou, 1986.

[4] Bruguières A., Cattaneo A., Keller B., Torossian C., Déformation, Quantification, Théorie de Lie, Panoramas et Synthèses, SMF, 2005.

[5] Bayen F., Flato M., Fronsdal C., Lichnerowicz A., Sternheimer D., Deformation theory and quantization. I. Deformations of symplectic structures, Ann. Physics 111 (1978), 61-110.

Bayen F., Flato M., Fronsdal C., Lichnerowicz A., Sternheimer D., Deformation theory and quantization. II. Physical applications, Ann. Physics 111 (1978), 111-151.

[6] Crawley-Boevey W., Holland M.P., Noncommutative deformations of Kleinian singularities, Duke Math. J. 92 (1998), 605-635.

[7] Chiang L., Chu H., Kang M.C., Generation of invariants, J. Algebra 221 (1999), 232-241.

[8] Fronsdal C., Kontsevich M., Quantization on curves, Lett. Math. Phys. 79 (2007), 109-129, math-ph/0507021.

[9] Gerstenhaber M., The cohomology structure of an associative ring, Ann. of Math. (2) 78 (1963), $267-288$.

[10] Guieu L., Roger C., avec un appendice de Sergiescu V., L'Algèbre et le Groupe de Virasoro: aspects géométriques et algébriques, généralisations, Publication du Centre de Recherches Mathématiques de Montréal, série "Monographies, notes de cours et Actes de conférences", PM28, 2007.

[11] Kontsevich M., Deformation quantization of Poisson manifolds. I, Preprint IHES, 1997, q-alg/9709040.

[12] Loday J.L., Cyclic homology, Springer-Verlag, Berlin, 1998.

[13] Pichereau A., Cohomologie de Poisson en dimension trois, C. R. Math. Acad. Sci. Paris 340 (2005), 151-154.

[14] Pichereau A., Poisson (co)homology and isolated singularities, J. Algebra 299 (2006), 747-777, math.QA/0511201.

[15] Rannou E., Saux-Picart P., Cours de calcul formel, partie II, éditions Ellipses, 2002.

[16] Roger C., Vanhaecke P., Poisson cohomology of the affine plane, J. Algebra 251 (2002), 448-460.

[17] Springer T.A., Invariant theory, Lecture Notes in Math., Vol. 585, Springer-Verlag, 1977.

[18] Van den Bergh M., Noncommutative homology of some three-dimensional quantum spaces, in Proceedings of Conference on Algebraic Geometry and Ring Theory in honor of Michael Artin, Part III (Antwerp, 1992), K-Theory 8 (1994), 213-230. 